\title{
Seidel Elements and Mirror Transformations for Toric Stacks
}

\author{
FENGLONG YOU
}

\begin{abstract}
We give a precise relation between the mirror transformation and the Seidel elements for weak Fano toric Deligne-Mumford stacks. Our result generalizes the corresponding result for toric varieties proved by González and Iritani [5]. The correction coefficients that we computed match with the instanton corrections from genus 0 open Gromov-Witten invariants for toric Calabi-Yau orbifolds in [3].
\end{abstract}

\section{Introduction}

In [5], González and Iritani gave a precise relation between the mirror map and the Seidel elements for a smooth projective weak Fano toric variety $X$. The goal of this paper is to generalize the main theorem of [5] to a smooth projective weak Fano toric Deligne-Mumford stack $\mathcal{X}$.

Let $\mathcal{X}$ be a smooth projective weak Fano toric Deligne-Mumford stack. The mirror theorem can be stated as an equality between the $I$-function and the $J$ function via a change of coordinates, called mirror map (or mirror transformation). We refer to [4] and Section 4.1 of [6] for further discussions.

Let $Y$ be a monotone symplectic manifold. For a loop $\lambda$ in the group of Hamiltonian symplectomorphisms on $Y$, Seidel [10] constructed an invertible element $S(\lambda)$ in (small) quantum cohomology counting sections of the associated Hamiltonian $Y$-bundle $E_{\lambda} \rightarrow \mathbb{P}^{1}$. The Seidel element $S(\lambda)$ defines an element in Aut $(Q H(Y))$ via quantum multiplication, and the map $\lambda \mapsto S(\lambda)$ gives a representation of $\pi_{1}(\operatorname{Ham}(Y))$ on $Q H(Y)$. McDuff and Tolman [9] extended this construction to all symplectic manifolds. The definition of Seidel representation and Seidel element were extended to symplectic orbifolds by Tseng and Wang [11].

Let $D_{1}, \ldots, D_{m}$ be the classes in $H^{2}(X)$ Poincaré dual to the toric divisors. When the loop $\lambda$ is a circle action, McDuff and Tolman [9] considered the Seidel element $\tilde{S}_{j}$ associated to an action $\lambda_{j}$ that fixes the toric divisor $D_{j}$. Given a circle action on $X$ (resp. $\mathcal{X}$ ), the Seidel element in [5] (resp. [11]) is defined using the small quantum cohomology ring. In this paper, we need to define it, for smooth projective Deligne-Mumford stack, with deformed quantum cohomology to include the bulk deformations. For weak Fano toric Deligne-Mumford stack, the mirror theorem in [6] shows that the mirror map $\tau(y) \in H_{\text {orb }}^{\leq 2}(\mathcal{X})$; therefore, we will only need bulk deformations with $\tau \in H_{\text {orb }}^{\leq 2}(\mathcal{X})$.

Received February 1, 2015. Revision received May 13, 2015. 
We consider the Seidel element $\tilde{S}_{j}$ associated to the toric divisor $D_{j}$ and the Seidel element $\tilde{S}_{m+j}$ corresponding to the box element $s_{j}$. The Seidel element in Definition 2.3 shows that $S=q_{0} \tilde{S}$ is a pull-back of a coefficient of the $J$-function $J_{\mathcal{E}_{j}}$ of the associated orbifiber bundle $\mathcal{E}_{j}$; hence, we can use the mirror theorem for $\mathcal{E}_{j}$ to calculate $\tilde{S}_{j}$ when $\mathcal{E}_{j}$ is weak Fano.

We extend the definition of the Batyrev element $\tilde{D}_{j}$ to weak Fano toric Deligne-Mumford stacks via partial derivatives of the mirror map $\tau(y)$. As analogues of the Seidel elements in B-model, the Batyrev elements can be explicitly computed from the $I$-function of $\mathcal{X}$. The following theorem states that the Seidel and Batyrev elements only differ by a multiplication of a correction function.

THeOREM 1.1. Let $X$ be a smooth projective toric Deligne-Mumford stack with $\rho^{S} \in \operatorname{cl}\left(C^{S}(\mathcal{X})\right)$.

(i) The Seidel element $\tilde{S}_{j}$ associated to the toric divisor $D_{j}$ is given by

$$
\tilde{S}_{j}(\tau(y))=\exp \left(-g_{0}^{(j)}(y)\right) \tilde{D}_{j}(y),
$$

where $\tau(y)$ is the mirror map of $\mathcal{X}$, and the function $g_{0}^{(j)}$ is given explicitly in (40);

(ii) The Seidel element $\tilde{S}_{m+j}$ corresponding to the box element $s_{j}$ is given by

$$
\tilde{S}_{m+j}(\tau(y))=\exp \left(-g_{0}^{(m+j)}\right) y^{-D_{m+j}^{S \vee}} \tilde{D}_{m+j}(y),
$$

where $\tau(y)$ is the mirror map of $\mathcal{X}$, and the function $g_{0}^{(m+j)}$ is given explicitly in (51).

It appears that the correction coefficients in the theorem coincide with the instanton corrections in Theorem 1.4 in [3]. This phenomenon also indicates that the deformed quantum cohomology ring of the toric Deligne-Mumford stack $\mathcal{X}$ is isomorphic to the Batyrev ring given in [6].

\section{Seidel Elements and $J$-Functions}

\subsection{Seidel Elements}

In this section, we fix our notation and construct the Seidel elements of smooth projective Deligne-Mumford stacks using $\tau$-deformed quantum cohomology.

Let $\mathcal{X}$ be a smooth projective Deligne-Mumford stack equipped with a $\mathbb{C}^{\times}$ action.

Definition 2.1. The associated orbifiber bundle of the $\mathbb{C}^{\times}$-action is the $\mathcal{X}$ bundle over $\mathbb{P}^{1}$

$$
\mathcal{E}:=\mathcal{X} \times\left(\mathbb{C}^{2} \backslash\{0\}\right) / \mathbb{C}^{\times} \rightarrow \mathbb{P}^{1},
$$

where $\mathbb{C}^{\times}$acts on $\mathbb{C}^{2} \backslash\{0\}$ via the standard diagonal action.

Let $\phi_{1}, \ldots, \phi_{N}$ be a basis for the orbifold cohomology ring $H_{\text {orb }}^{*}(\mathcal{X}):=$ $H^{*}(\mathcal{I} \mathcal{X} ; \mathbb{Q})$ of $\mathcal{X}$, where $\mathcal{I} \mathcal{X}$ is the inertia stack of $\mathcal{X}$. Let $\phi^{1}, \ldots, \phi^{N}$ be the dual 
basis of $\phi_{1}, \ldots, \phi_{N}$ with respect to the orbifold Poincaré pairing. Furthermore, let $\hat{\phi}_{1}, \ldots, \hat{\phi}_{M}$ denote a basis for the orbifold cohomology $H_{\text {orb }}^{*}(\mathcal{E}):=H^{*}(\mathcal{I} \mathcal{E} ; \mathbb{Q})$ of $\mathcal{E}$. Let $\hat{\phi}^{1}, \ldots, \hat{\phi}^{M}$ be the dual basis of $\hat{\phi}_{1}, \ldots, \hat{\phi}_{M}$ with respect to the orbifold Poincaré pairing.

We will use $X$ to denote the coarse moduli space of $\mathcal{X}$ and use $E$ to denote the coarse moduli space of $\mathcal{E}$. Then the $\mathbb{C}^{\times}$action on $\mathcal{X}$ descends to the $\mathbb{C}^{\times}$ action on $X$ with $E$ being the associated bundle. Following [8] and [5], there is a (noncanonical) splitting

$$
H^{*}(\mathcal{E} ; \mathbb{Q}) \cong H^{*}(E ; \mathbb{Q}) \cong H^{*}(X ; \mathbb{Q}) \otimes H^{*}\left(\mathbb{P}^{1} ; \mathbb{Q}\right) \cong H^{*}(\mathcal{X} ; \mathbb{Q}) \otimes H^{*}\left(\mathbb{P}^{1} ; \mathbb{Q}\right) \text {. }
$$

According to [5], there is a unique $\mathbb{C}^{\times}$-fixed component $F_{\max } \subset X^{\mathbb{C}^{\times}}$such that the normal bundle of $F_{\max }$ has only negative $\mathbb{C}^{\times}$-weights. Let $\sigma_{0}$ be the section associated to a fixed point in $F_{\max }$. Following [5], there is a splitting defined by this maximal section,

$$
\begin{aligned}
H_{2}(\mathcal{E} ; \mathbb{Z}) / \text { tors } & \cong H_{2}(E ; \mathbb{Z}) / \text { tors } \cong \mathbb{Z}\left[\sigma_{0}\right] \oplus\left(H_{2}(X, \mathbb{Z}) / \text { tors }\right) \\
& \cong \mathbb{Z}\left[\sigma_{0}\right] \oplus\left(H_{2}(\mathcal{X}, \mathbb{Z}) / \text { tors }\right) .
\end{aligned}
$$

Let $\mathrm{NE}(X) \subset H_{2}(X ; \mathbb{R})$ denote the Mori cone, that is, the cone generated by effective curves, and set

$$
\mathrm{NE}(X)_{\mathbb{Z}}:=\mathrm{NE}(X) \cap\left(H_{2}(X, \mathbb{Z}) / \text { tors }\right) .
$$

Then, by Lemma 2.2 of [5] we have

$$
\mathrm{NE}(E)_{\mathbb{Z}}=\mathbb{Z}_{\geq 0}\left[\sigma_{0}\right]+\mathrm{NE}(X)_{\mathbb{Z}}
$$

Let $H_{2}^{\mathrm{sec}}(E ; \mathbb{Z})$ be the affine subspace of $H_{2}(E, \mathbb{Z}) /$ tors that consists of the classes that project to the positive generator of $H_{2}\left(\mathbb{P}^{1} ; \mathbb{Z}\right)$. Setting

$$
\mathrm{NE}(E)_{\mathbb{Z}}^{\mathrm{sec}}:=\mathrm{NE}(E)_{\mathbb{Z}} \cap H_{2}^{\mathrm{sec}}(E ; \mathbb{Z}),
$$

we obtain

$$
\mathrm{NE}(E)_{\mathbb{Z}}^{\mathrm{sec}}=\left[\sigma_{0}\right]+\mathrm{NE}(X)_{\mathbb{Z}} .
$$

We choose a nef integral basis $\left\{p_{1}, \ldots, p_{r}\right\}$ of $H^{2}(\mathcal{X} ; \mathbb{Q})$; then there are unique lifts of $p_{1}, \ldots, p_{r}$ in $H^{2}(\mathcal{E} ; \mathbb{Q})$ that vanish on $\left[\sigma_{0}\right]$. By abuse of notation, we also denote these lifts as $p_{1}, \ldots, p_{r}$; these lifts are also nef. Let $p_{0}$ be the pullback of the positive generator of $H^{2}\left(\mathbb{P}^{1} ; \mathbb{Z}\right)$ in $H^{2}(\mathcal{E} ; \mathbb{Q})$. Therefore, $\left\{p_{0}, p_{1}, \ldots, p_{r}\right\}$ is an integral basis of $H^{2}(\mathcal{E} ; \mathbb{Q})$.

Let $q_{0}, q_{1}, \ldots, q_{r}$ be the Novikov variables of $\mathcal{E}$ dual to $p_{0}, p_{1}, \ldots, p_{r}$, and $q_{1}, \ldots, q_{r}$ be the Novikov variables of $\mathcal{X}$ dual to $p_{1}, \ldots, p_{r}$. We denote the Novikov ring of $\mathcal{X}$ and the Novikov ring of $\mathcal{E}$ by

$$
\Lambda_{\mathcal{X}}:=\mathbb{Q}\left[\left[q_{1}, \ldots, q_{r}\right]\right] \text { and } \Lambda_{\mathcal{E}}:=\mathbb{Q}\left[\left[q_{0}, q_{1}, \ldots, q_{r}\right]\right],
$$

respectively. For each $d \in \mathrm{NE}(X)_{\mathbb{Z}}$, we write

$$
q^{d}:=q_{1}^{\left\langle p_{1}, d\right\rangle} \cdots q_{r}^{\left\langle p_{r}, d\right\rangle} \in \Lambda_{\mathcal{X}},
$$

and for each $\beta \in \mathrm{NE}(E)_{\mathbb{Z}}$, we write

$$
q^{\beta}:=q_{0}^{\left\langle p_{0}, \beta\right\rangle} q_{1}^{\left\langle p_{1}, \beta\right\rangle} \cdots q_{r}^{\left\langle p_{r}, \beta\right\rangle} \in \Lambda_{\mathcal{E}} .
$$


The $\tau$-deformed orbifold quantum product is defined as follows:

$$
\alpha \bullet_{\tau} \beta=\sum_{d \in \operatorname{NE}(X)_{\mathbb{Z}}} \sum_{l \geq 0} \sum_{k=1}^{N} \frac{1}{l !}\left\langle\alpha, \beta, \tau, \ldots, \tau, \phi_{k}\right\rangle_{0, l+3, d}^{\mathcal{X}} q^{d} \phi^{k} ;
$$

the associated quantum cohomology ring is denoted by

$$
Q H_{\tau}(\mathcal{X}):=\left(H(\mathcal{X}) \otimes_{\mathbb{Q}} \Lambda_{\mathcal{X}}, \bullet_{\tau}\right)
$$

Notation 2.2. For a smooth projective Deligne-Mumford stack $\mathcal{X}$, we denote by $H_{t w}^{\leq 2}(\mathcal{X})$ the complementary subspace of $H^{2}(\mathcal{X})$ in $H_{\text {orb }}^{\leq 2}(\mathcal{X})$ supported on the twisted sectors, that is, we have the decomposition

$$
H_{\text {orb }}^{\leq 2}(\mathcal{X})=H^{2}(\mathcal{X}) \oplus H_{t w}^{\leq 2}(\mathcal{X})
$$

Definition 2.3. The Seidel element of $\mathcal{X}$ is the class

$$
S(\hat{\tau}):=\sum_{\alpha} \sum_{\beta \in \operatorname{NE}(E)_{\mathbb{Z}}^{\sec }} \sum_{l \geq 0} \frac{1}{l !}\left\langle\mathbf{1}, \hat{\tau}_{t w}, \ldots, \hat{\tau}_{t w}, l_{*} \phi_{\alpha} \psi\right\rangle_{0, l+2, \beta}^{\mathcal{E}} \phi^{\alpha} e^{\left\langle\hat{\tau}_{0,2}, \beta\right\rangle}
$$

in $Q H_{\tau}(\mathcal{X}) \otimes_{\Lambda_{\mathcal{X}}} \Lambda_{\mathcal{E}}$. Here $\imath: \mathcal{X} \rightarrow \mathcal{E}$ is the inclusion of a fiber, and

$$
l_{*}: H^{*}(\mathcal{I} \mathcal{X} ; \mathbb{Q}) \rightarrow H^{*+2}(\mathcal{I} \mathcal{E} ; \mathbb{Q})
$$

is the Gysin map. Moreover,

$$
e^{\left\langle\hat{\tau}_{0,2}, \beta\right\rangle}=q^{\beta}=q_{0}^{\left\langle p_{0}, \beta\right\rangle} \cdots q_{r}^{\left\langle p_{r}, \beta\right\rangle},
$$

where

$$
\hat{\tau}_{0,2}=\sum_{a=0}^{r} p_{a} \log q_{a} \in H^{2}(\mathcal{E})
$$

and

$$
\hat{\tau}=\hat{\tau}_{0,2}+\hat{\tau}_{t w} \in H^{2}(\mathcal{E}) \oplus H_{t w}^{\leq 2}(\mathcal{E})=H_{\text {orb }}^{\leq 2}(\mathcal{E}) .
$$

The Seidel element can be factorized as

$$
S(\hat{\tau})=q_{0} \tilde{S}(\hat{\tau}) \quad \text { with } \tilde{S}(\hat{\tau}) \in Q H_{\tau}(\mathcal{X}) .
$$

REMARK 2.4. The descendant class $\psi$ in equation (5) can be eliminated by the string equation; hence, our definition of Seidel elements matches with the definition in [5]

\subsection{J-Functions}

We will explain the relation between the Seidel element and the $J$-function of the associated bundle $\mathcal{E}$. 
Definition 2.5. The $J$-function of $\mathcal{E}$ is the cohomology-valued function

$$
\begin{aligned}
J_{\mathcal{E}}(\hat{\tau}, z)= & e^{\hat{\tau}_{0,2} / z}\left(1+\sum_{\alpha} \sum_{(\beta, l) \neq(0,0), \beta \in \mathrm{NE}(E)_{\mathbb{Z}}} \frac{e^{\left\langle\hat{\tau}_{0,2}, \beta\right\rangle}}{l !}\right. \\
& \times\left\langle\mathbf{1}, \hat{\tau}_{t w}, \ldots, \hat{\tau}_{t w},\left.\frac{\hat{\phi}_{\alpha}}{z-\psi}\right|_{0, l+2, \beta} ^{\mathcal{E}} \hat{\phi}^{\alpha}\right),
\end{aligned}
$$

where $\hat{\phi}_{\alpha} /(z-\psi)=\sum_{n \geq 0} z^{-1-n} \hat{\phi}_{\alpha} \psi^{n}$.

Note that when $n=0$, we have

(i) $\sum_{\alpha}\left\langle\mathbf{1}, \hat{\tau}_{t w}, \ldots, \hat{\tau}_{t w}, \hat{\phi}_{\alpha}\right\rangle_{0, l+2, \beta}^{\mathcal{E}} \hat{\phi}^{\alpha}=0$ for $(l, \beta) \neq(1,0)$;

(ii) $\sum_{\alpha}\left\langle\mathbf{1}, \hat{\tau}_{t w}, \ldots, \hat{\tau}_{t w}, \hat{\phi}_{\alpha}\right\rangle_{0, l+2, \beta}^{\mathcal{E}} \hat{\phi}^{\alpha}=\hat{\tau}_{t w}$ for $(l, \beta)=(1,0)$.

The $J$-function can be expanded in terms of powers of $z^{-1}$ as follows:

$$
\begin{aligned}
J_{\mathcal{E}}(\hat{\tau}, z)= & e^{\sum_{a=0}^{r} p_{a} \log q_{a} / z}\left(1+z^{-1} \hat{\tau}_{t w}\right. \\
& \left.+z^{-2} \sum_{n=0}^{\infty} F_{n}\left(q_{1}, \ldots, q_{r} ; \hat{\tau}\right) q_{0}^{n}+O\left(z^{-3}\right)\right),
\end{aligned}
$$

where

$$
\begin{aligned}
& F_{n}\left(q_{1}, \ldots, q_{r} ; \hat{\tau}\right) \\
& \quad=\sum_{\alpha=1}^{M} \sum_{d \in \operatorname{NE}(X)_{\mathbb{Z}}} \sum_{l \geq 0} \frac{1}{l !}\left\langle\mathbf{1}, \hat{\tau}_{t w}, \ldots, \hat{\tau}_{t w}, \hat{\phi}_{\alpha} \psi\right\rangle_{0, l+2, d+n \sigma_{0}}^{\mathcal{E}} q^{d} \hat{\phi}^{\alpha} .
\end{aligned}
$$

Proposition 2.6. The Seidel element corresponding to the $\mathbb{C}^{\times}$action on $\mathcal{X}$ is given by

$$
S(\hat{\tau})=\imath^{*}\left(F_{1}\left(q_{1}, \ldots, q_{r} ; \hat{\tau}\right) q_{0}\right) .
$$

Proof. The proof here is identical to the proof given in Proposition 2.5 of [5] for smooth projective varieties:

Using the duality identity

$$
\sum_{\alpha=1}^{M} \hat{\phi}_{\alpha} \otimes \imath^{*} \hat{\phi}^{\alpha}=\sum_{\alpha=1}^{N} l_{*} \phi_{\alpha} \otimes \phi^{\alpha},
$$

we can see that

$$
\begin{aligned}
& \iota^{*} F_{1}\left(q_{1}, \ldots, q_{r} ; \hat{\tau}\right) \\
& \quad=\sum_{\alpha=1}^{N} \sum_{d \in \operatorname{NE}(X)_{\mathbb{Z}}} \sum_{l \geq 0} \frac{1}{l !}\left\langle\mathbf{1}, \hat{\tau}_{t w}, \ldots, \hat{\tau}_{t w}, l_{*} \phi_{\alpha} \psi\right\rangle_{0, l+2, d+\sigma_{0}}^{\mathcal{E}} q^{d} \phi^{\alpha} .
\end{aligned}
$$

Hence, the conclusion follows, that is,

$$
S(\hat{\tau})=\iota^{*}\left(F_{1}\left(q_{1}, \ldots, q_{r} ; \hat{\tau}\right) q_{0}\right) .
$$




\section{Seidel Elements Corresponding to Toric Divisors}

\subsection{A Review of Toric Deligne-Mumford Stacks}

In this section, we define toric Deligne-Mumford stacks following the construction of [2] and [6].

A toric Deligne-Mumford stack is defined by a stacky fan $\Sigma=(\mathbf{N}, \Sigma, \beta)$, where $\mathbf{N}$ is a finitely generated Abelian group, $\Sigma \subset \mathbf{N}_{\mathbb{Q}}=\mathbf{N} \otimes_{\mathbb{Z}} \mathbb{Q}$ is a rational simplicial fan, and $\beta: \mathbb{Z}^{m} \rightarrow \mathbf{N}$ is a homomorphism. We assume that $\beta$ has finite cokernel and the rank of $\mathbf{N}$ is $n$. The map $\mathbb{Z}^{m} \stackrel{\beta}{\longrightarrow} \mathbf{N} \rightarrow \mathbf{N}_{\mathbb{Q}}$ generates the 1skeleton of the fan $\Sigma$. Let $b_{i}$ be the image under $\beta$ of the standard basis of $\mathbb{Z}^{m}$, and $\overline{b_{i}}$ be the image of $b_{i}$ under the canonical map $\mathbf{N} \rightarrow \mathbf{N}_{\mathbb{Q}}$. Let $\mathbb{L} \subset \mathbb{Z}^{m}$ be the kernel of $\beta$. Then the fan sequence is the following exact sequence:

$$
0 \longrightarrow \mathbb{L} \longrightarrow \mathbb{Z}^{m} \stackrel{\beta}{\longrightarrow} \mathbf{N}
$$

Let $\beta^{\vee}:\left(\mathbb{Z}^{*}\right)^{m} \rightarrow \mathbb{L}^{\vee}$ be the Gale dual of $\beta$ in [2], where $\mathbb{L}^{\vee}:=H^{1}\left(\operatorname{Cone}(\beta)^{*}\right)$ is an extension of $\mathbb{L}^{*}=\operatorname{Hom}(\mathbb{L}, \mathbb{Z})$ by a torsion subgroup. The divisor sequence is the following exact sequence:

$$
0 \longrightarrow \mathbf{N}^{*} \stackrel{\beta^{*}}{\longrightarrow}\left(\mathbb{Z}^{*}\right)^{m} \stackrel{\beta^{\vee}}{\longrightarrow} \mathbb{L}^{\vee} .
$$

By applying $\operatorname{Hom}_{\mathbb{Z}}\left(-, \mathbb{C}^{\times}\right)$to the dual map $\beta^{\vee}$ we have a homomorphism

$$
\alpha: G \rightarrow\left(\mathbb{C}^{\times}\right)^{m}, \quad \text { where } G:=\operatorname{Hom}_{\mathbb{Z}}\left(\mathbb{L}^{\vee}, \mathbb{C}^{\times}\right),
$$

and we let $G$ act on $\mathbb{C}^{m}$ via this homomorphism.

The collection of anticones $\mathcal{A}$ is defined as follows:

$$
\mathcal{A}:=\left\{I: \sum_{i \notin I} \mathbb{R}_{\geq 0} \bar{b}_{i} \in \Sigma\right\} .
$$

Let $\mathcal{U}$ denote the open subset of $\mathbb{C}^{m}$ defined by $\mathcal{A}$ :

$$
\mathcal{U}:=\mathbb{C}^{m} \backslash \bigcup_{I \notin \mathcal{A}} \mathbb{C}^{I},
$$

where

$$
\mathbb{C}^{I}=\left\{\left(z_{1}, \ldots, z_{m}\right): z_{i}=0 \text { for } i \notin I\right\} .
$$

Definition 3.1. Following [6], the toric Deligne-Mumford stack $\mathcal{X}$ is defined as the quotient stack

$$
\mathcal{X}:=[\mathcal{U} / G]
$$

REMARK 3.2. The toric variety $X$ associated to the fan $\Sigma$ is the coarse moduli space of $\mathcal{X}([2])$.

Definition 3.3 ([6]). Given a stacky fan $\Sigma=(\mathbf{N}, \Sigma, \beta)$, we define the set of box elements $\operatorname{Box}(\boldsymbol{\Sigma})$ as follows:

$$
\operatorname{Box}(\Sigma)=:\left\{v \in \mathbf{N}: \bar{v}=\sum_{k \notin I} c_{k} \bar{b}_{k} \text { for some } 0 \leq c_{k}<1, I \in \mathcal{A}\right\} \text {. }
$$


We assume that $\Sigma$ is complete; then the connected components of the inertia stack $\mathcal{I} \mathcal{X}$ are indexed by the elements of $\operatorname{Box}(\boldsymbol{\Sigma})$ (see [2]). Moreover, given $v \in$ $\operatorname{Box}(\Sigma)$, the age of the corresponding connected component of $\mathcal{I} \mathcal{X}$ is defined by $\operatorname{age}(v):=\sum_{k \notin I} c_{k}$.

The Picard group $\operatorname{Pic}(\mathcal{X})$ of $\mathcal{X}$ can be identified with the character group $\operatorname{Hom}\left(G, \mathbb{C}^{\times}\right)$. Hence,

$$
\mathbb{L}^{\vee}=\operatorname{Hom}\left(G, \mathbb{C}^{\times}\right) \cong \operatorname{Pic}(\mathcal{X}) \cong H^{2}(\mathcal{X} ; \mathbb{Z})
$$

We can also use the extended stacky fans introduced by Jiang [7] to define the toric Deligne-Mumford stacks. Given a stacky fan $\Sigma=(\mathbf{N}, \Sigma, \beta)$ and a finite set

$$
S=\left\{s_{1}, \ldots, s_{l}\right\} \subset \mathbf{N}_{\Sigma}:=\{c \in \mathbf{N}: \bar{c} \in|\Sigma|\},
$$

the $S$-extended stacky fan is given by $\left(\mathbf{N}, \Sigma, \beta^{S}\right)$, where $\beta^{S}: \mathbb{Z}^{m+l} \rightarrow \mathbf{N}$ is defined by

$$
\beta^{S}\left(e_{i}\right)= \begin{cases}b_{i}, & 1 \leq i \leq m \\ s_{i-m}, & m+1 \leq i \leq m+l .\end{cases}
$$

Let $\mathbb{L}^{S}$ be the kernel of $\beta^{S}: \mathbb{Z}^{m+l} \rightarrow \mathbf{N}$. Then we have the following $S$-extended fan sequence:

$$
0 \longrightarrow \mathbb{L}^{S} \longrightarrow \mathbb{Z}^{m+l} \stackrel{\beta^{S}}{\longrightarrow} \mathbf{N}
$$

By the Gale duality we have the $S$-extended divisor sequence

$$
0 \longrightarrow \mathbf{N}^{*} \stackrel{\beta^{*}}{\longrightarrow}\left(\mathbb{Z}^{*}\right)^{m+l} \stackrel{\beta^{S \vee}}{\longrightarrow} \mathbb{L}^{S \vee},
$$

where $\mathbb{L}^{S \vee}:=H^{1}\left(\operatorname{Cone}\left(\beta^{S}\right)^{*}\right)$.

Assumption 3.4. In the rest of the paper, we assume that the set

$$
\{v \in \operatorname{Box}(\Sigma) ; \operatorname{age}(v) \leq 1\} \cup\left\{b_{1}, \ldots, b_{m}\right\}
$$

generates $\mathbf{N}$ over $\mathbb{Z}$, and we choose the set

$$
S=\left\{s_{1}, \ldots, s_{l}\right\} \subset \operatorname{Box}(\Sigma)
$$

such that the set $\left\{b_{1}, \ldots, b_{m}, s_{1}, \ldots, s_{l}\right\}$ generates $\mathbf{N}$ over $\mathbb{Z}$ and age $\left(s_{j}\right) \leq 1$ for $1 \leq j \leq l$.

Let $D_{i}^{S}$ be the image of the standard basis of $\left(\mathbb{Z}^{*}\right)^{m+l}$ under the map $\beta^{S \vee}$. Then there is a canonical isomorphism

$$
\mathbb{L}^{S \vee} \otimes \mathbb{Q} \cong\left(\mathbb{L}^{\vee} \otimes \mathbb{Q}\right) \oplus \bigoplus_{i=m+1}^{m+l} \mathbb{Q} D_{i}^{S},
$$

which can be constructed as follows [6].

Since $\Sigma$ is complete, for $m<j \leq m+l$, the box element $s_{j-m}$ is contained in some cone in $\Sigma$. Namely,

$$
s_{j-m}=\sum_{i \notin I_{j}^{S}} c_{j i} b_{i} \quad \text { in } \mathbf{N} \otimes \mathbb{Q}, c_{j i} \geq 0, \exists I_{j}^{S} \in \mathcal{A}^{S},
$$


where $I_{j}^{S}$ is the "anticone" of the cone containing $s_{j-m}$.

By the $S$-extended fan sequence (15) tensored with $\mathbb{Q}$, we have the following short exact sequence:

$$
0 \longrightarrow \mathbb{L}^{S} \otimes \mathbb{Q} \longrightarrow \mathbb{Q}^{m+l} \stackrel{\beta^{S}}{\longrightarrow} \mathbf{N} \otimes \mathbb{Q} \longrightarrow 0 .
$$

Hence, there exists a unique $D_{j}^{S \vee} \in \mathbb{L}^{S} \otimes \mathbb{Q}$ such that

$$
\left\langle D_{i}^{S}, D_{j}^{S \vee}\right\rangle= \begin{cases}1, & i=j ; \\ -c_{j i}, & i \notin I_{j}^{S} ; \\ 0, & i \in I_{j}^{S} \backslash\{j\} .\end{cases}
$$

These vectors $D_{j}^{S \vee}$ define the decomposition

$$
\mathbb{L}^{S \vee} \otimes \mathbb{Q}=\operatorname{Ker}\left(\left(D_{m+1}^{S \vee}, \ldots, D_{m+l}^{S \vee}\right): \mathbb{L}^{S \vee} \otimes \mathbb{Q} \rightarrow \mathbb{Q}^{l}\right) \oplus \bigoplus_{j=m+1}^{m+l} \mathbb{Q}_{j}^{S} .
$$

We identify the first factor $\operatorname{Ker}\left(D_{m+1}^{S \vee}, \ldots, D_{m+l}^{S \vee}\right)$ with $\mathbb{L}^{\vee} \otimes \mathbb{Q}$. Via this decomposition, we can regard $H^{2}(\mathcal{X}, \mathbb{Q}) \cong \mathbb{L}^{\vee} \otimes \mathbb{Q}$ as a subspace of $\mathbb{L}^{S \vee} \otimes \mathbb{Q}$.

Let $D_{i}$ be the image of $D_{i}^{S}$ in $\mathbb{L}^{\vee} \otimes \mathbb{Q}$ under this decomposition. Then

$$
D_{i}=0 \text { for } m+1 \leq i \leq m+l .
$$

Let $\mathcal{A}^{S}$ be the collection of $S$-extended anti-cones, that is,

$$
\mathcal{A}^{S}:=\left\{I^{S}: \sum_{i \notin I^{S}} \mathbb{R}_{\geq 0} \overline{\beta^{S}\left(e_{i}\right)} \in \Sigma\right\} .
$$

Note that

$$
\left\{s_{1}, \ldots, s_{l}\right\} \subset I^{S}, \quad \forall I^{S} \in \mathcal{A}^{S} .
$$

By applying $\operatorname{Hom}_{\mathbb{Z}}\left(-, \mathbb{C}^{\times}\right)$to the $S$-extended dual map $\beta^{\vee}$ we have a homomorphism

$$
\alpha^{S}: G^{S} \rightarrow\left(\mathbb{C}^{\times}\right)^{m+l}, \quad \text { where } G^{S}:=\operatorname{Hom}_{\mathbb{Z}}\left(\mathbb{L}^{S \vee}, \mathbb{C}^{\times}\right) .
$$

We define $\mathcal{U}$ to be the open subset of $\mathbb{C}^{m+l}$ defined by $\mathcal{A}^{S}$ :

$$
\mathcal{U}^{S}:=\mathbb{C}^{m+l} \backslash \bigcup_{I^{S} \notin \mathcal{A}^{S}} \mathbb{C}^{I^{S}}=\mathcal{U} \times\left(\mathbb{C}^{\times}\right)^{l}
$$

where

$$
\mathbb{C}^{I^{S}}=\left\{\left(z_{1}, \ldots, z_{m+l}\right): z_{i}=0 \text { for } i \notin I^{S}\right\} .
$$

Let $G^{S}$ act on $\mathcal{U}^{S}$ via $\alpha^{S}$. Then we obtain the quotient stack $\left[\mathcal{U}^{S} / G^{S}\right]$. Jiang [7] showed that

$$
\left[\mathcal{U}^{S} / G^{S}\right] \cong[\mathcal{U} / G]=\mathcal{X}
$$




\subsection{Mirror Theorem for Toric Stacks}

Coates et al. [4] defined the $S$-extended $I$-function of a smooth toric DeligneMumford stack $\mathcal{X}$ with semiprojective coarse moduli space and proved that this $I$-function is a point of Givental's Lagrangian cone $\mathcal{L}$ for the Gromov-Witten theory of $\mathcal{X}$. In this paper, we only need this theorem for the weak Fano case. In this case, the mirror theorem will take a particularly simple form, which can be stated as an equality of $I$-function and $J$-function via a change of variables, called mirror map.

To state the mirror theorem for weak Fano toric Deligne-Mumford stack, we need the following definitions.

We define the $S$-extended Kähler cone $C_{\mathcal{X}}^{S}$ as

$$
C_{\mathcal{X}}^{S}:=\bigcap_{I^{S} \in \mathcal{A}^{S}} \Sigma_{i \in I^{S}} \mathbb{R}_{>0} D_{i}^{S}
$$

and the Kähler cone $C_{\mathcal{X}}$ as

$$
C_{\mathcal{X}}:=\bigcap_{I \in \mathcal{A}} \Sigma_{i \in I} \mathbb{R}_{>0} D_{i} .
$$

Let $p_{1}^{S}, \ldots, p_{r+l}^{S}$ be an integral basis of $\mathbb{L}^{S \vee}$, where $r=m-n$, such that $p_{i}^{S}$ is in the closure $\operatorname{cl}\left(C_{\mathcal{X}}^{S}\right)$ of the $S$-extended Kähler cone $C_{\mathcal{X}}^{S}$ for all $1 \leq i \leq r+l$ and $p_{r+1}^{S}, \ldots, p_{r+l}^{S}$ are in $\sum_{i=m+1}^{m+l} \mathbb{R}_{\geq 0} D_{i}^{S}$. We denote the image of $p_{i}^{S}$ in $\mathbb{L}^{\vee} \otimes \mathbb{R}$ by $p_{i}$; therefore, $p_{1}, \ldots, p_{r}$ are nef, and $p_{r+1}, \ldots, p_{r+l}$ are zero. We define the matrix $\left(m_{i a}\right)$ by

$$
D_{i}^{S}=\sum_{a=1}^{r+l} m_{i a} p_{a}^{S}, \quad m_{i a} \in \mathbb{Z} .
$$

Then the class $D_{i}$ of toric divisor is given by

$$
D_{i}=\sum_{a=1}^{r} m_{i a} p_{a}
$$

Definition 3.5 ([6], Sect. 3.1.4). A toric Deligne-Mumford stack $\mathcal{X}$ is called weak Fano if the first Chern class $\rho$ satisfies

$$
\rho=c_{1}(T \mathcal{X})=\sum_{i=1}^{m} D_{i} \in \operatorname{cl}(C \mathcal{X}),
$$

where $C_{\mathcal{X}}$ is the Kähler cone of $\mathcal{X}$.

We will need a slightly stronger condition:

$$
\rho^{S}:=D_{1}^{S}+\cdots+D_{m+l}^{S} \in \operatorname{cl}\left(C_{\mathcal{X}}^{S}\right),
$$

where $C_{\mathcal{X}}^{S}$ is the $S$-extended Kähler cone. By Lemma 3.3 of [6] we can see that $\rho^{S} \in \operatorname{cl}\left(C_{\mathcal{X}}^{S}\right)$ implies $\rho \in \operatorname{cl}\left(C_{\mathcal{X}}\right)$. Moreover, under Assumption 3.4, we have

$$
\rho^{S} \in \operatorname{cl}\left(C_{\mathcal{X}}^{S}\right) \quad \text { if and only if } \rho \in \operatorname{cl}\left(C_{\mathcal{X}}\right)
$$


For a real number $r$, let $\lceil r\rceil,\lfloor r\rfloor$, and $\{r\}$ be the ceiling, floor, and fractional part of $r$, respectively.

Definition 3.6. We define two subsets $\mathbb{K}$ and $\mathbb{K}_{\mathrm{eff}}$ of $\mathbb{L}^{S} \otimes \mathbb{Q}$ as follows:

$$
\begin{aligned}
\mathbb{K} & :=\left\{d \in \mathbb{L}^{S} \otimes \mathbb{Q} ;\left\{i \in\{1, \ldots, m+l\} ;\left\langle D_{i}^{S}, d\right\rangle \in \mathbb{Z}\right\} \in \mathcal{A}^{S}\right\}, \\
\mathbb{K}_{\mathrm{eff}} & :=\left\{d \in \mathbb{L}^{S} \otimes \mathbb{Q} ;\left\{i \in\{1, \ldots, m+l\} ;\left\langle D_{i}^{S}, d\right\rangle \in \mathbb{Z}_{\geq 0}\right\} \in \mathcal{A}^{S}\right\} .
\end{aligned}
$$

REMARK 3.7. We use $\mathbb{K}_{\mathcal{E}_{j}}$ and $\mathbb{K}_{\mathrm{eff}, \mathcal{E}_{j}}$ to denote the corresponding sets for the associated bundle $\mathcal{E}_{j}$ and use $\mathbb{K}_{\mathcal{X}}$ and $\mathbb{K}_{\text {eff, } \mathcal{X}}$ to denote the corresponding sets for $\mathcal{X}$.

Definition 3.8 ([6], Sect. 3.1.3). The reduction function $v$ is defined as follows:

$$
\begin{aligned}
v: \mathbb{K} & \longrightarrow \operatorname{Box}(\boldsymbol{\Sigma}), \\
d & \longmapsto \sum_{i=1}^{m}\left\lceil\left\langle D_{i}^{S}, d\right\rangle\right\rceil b_{i}+\sum_{j=1}^{l}\left\lceil\left\langle D_{m+j}^{S}, d\right\rangle\right\rceil s_{j} .
\end{aligned}
$$

By the $S$-extended fan exact sequence we have

$$
\sum_{i=1}^{m}\left\langle D_{i}^{S}, d\right\rangle b_{i}+\sum_{j=1}^{l}\left\langle D_{m+j}^{S}, d\right\rangle s_{j}=0 \in \mathbf{N} \otimes \mathbb{Q} .
$$

Moreover, by the definition of $\mathbb{K}$ we have

$$
\left\langle D_{m+j}^{S}, d\right\rangle \in \mathbb{Z} \quad \text { for all } d \in \mathbb{K} \text { and } 1 \leq j \leq l .
$$

Hence,

$$
v(d)=\sum_{i=1}^{m}\left\{-\left\langle D_{i}^{S}, d\right\rangle\right\} b_{i}+\sum_{j=1}^{l}\left\{-\left\langle D_{m+j}^{S}, d\right\rangle\right\} s_{j}=\sum_{i=1}^{m}\left\{-\left\langle D_{i}^{S}, d\right\rangle\right\} b_{i} .
$$

The corresponding inertia component $\mathcal{X}_{v(d)}$ is given by

$$
\mathcal{X}_{v(d)}:=\left\{\left[z_{1}, \ldots, z_{m+l}\right] \in \mathcal{X} \mid z_{i}=0 \text { if }\left\langle D_{i}^{S}, d\right\rangle \notin \mathbb{Z}\right\},
$$

and the unit class of $H^{*}\left(\mathcal{X}_{v(d)}\right)$ is denoted by $\mathbf{1}_{v(d)}$.

By abuse of notation, we use $D_{i}$ to denote the divisor $\left\{z_{i}=0\right\} \subset \mathcal{X}$ and the cohomology class in $H^{2}(\mathcal{X} ; \mathbb{Z}) \cong \mathbb{L}^{\vee}$ for $1 \leq i \leq m$.

We consider the $\mathbb{C}^{\times}$-action fixing a toric divisor $D_{j}, 1 \leq j \leq m$; the action of $\mathbb{C}^{\times}$on $\mathbb{C}^{m}$ is given by

$$
\left(z_{1}, \ldots, z_{m}\right) \mapsto\left(z_{1}, \ldots, t^{-1} z_{j}, \ldots, z_{m}\right), \quad t \in \mathbb{C}^{\times} .
$$

We can extend this to the diagonal $\mathbb{C}^{\times}$-action on $\mathcal{U} \times\left(\mathbb{C}^{2} \backslash\{0\}\right)$ by

$$
\left(z_{1}, \ldots, z_{m}, u, v\right) \mapsto\left(z_{1}, \ldots, t^{-1} z_{j}, \ldots, z_{m}, t u, t v\right), \quad t \in \mathbb{C}^{\times} .
$$

The associated bundle $\mathcal{E}_{j}$ of the $\mathbb{C}^{\times}$-action on $\mathcal{X}$ is given by

$$
\mathcal{E}_{j}=\mathcal{U} \times\left(\mathbb{C}^{2} \backslash\{0\}\right) / G \times \mathbb{C}^{\times}
$$


We can also use the $S$-extended stacky fan of $\mathcal{X}$ to define $\mathcal{E}_{j}$ :

$$
\mathcal{E}_{j}=\mathcal{U}^{\mathcal{S}} \times\left(\mathbb{C}^{2} \backslash\{0\}\right) / G^{S} \times \mathbb{C}^{\times} .
$$

Therefore, $\mathcal{E}_{j}$ is also a toric Deligne-Mumford stack. We can identify $H^{2}\left(\mathcal{E}_{j} ; \mathbb{Z}\right)$ with the lattice of the characters of $G \times \mathbb{C}^{\times}$:

$$
H^{2}\left(\mathcal{E}_{j} ; \mathbb{Z}\right) \cong \mathbb{L}^{\vee} \oplus \mathbb{Z} \cong H^{2}(\mathcal{X} ; \mathbb{Z}) \oplus \mathbb{Z}
$$

Moreover, we have the divisor sequence

$$
0 \rightarrow \mathbf{N}^{*} \oplus \mathbb{Z} \rightarrow\left(\mathbb{Z}^{*}\right)^{m+2} \rightarrow \mathbb{L}^{\vee} \oplus \mathbb{Z}
$$

and the $S$-extended divisor sequence

$$
0 \rightarrow \mathbf{N}^{*} \oplus \mathbb{Z} \rightarrow\left(\mathbb{Z}^{*}\right)^{m+l+2} \rightarrow \mathbb{L}^{S \vee} \oplus \mathbb{Z} .
$$

Let $\hat{D}_{i}^{S}$ be the image of the standard basis of $\left(\mathbb{Z}^{*}\right)^{m+l+2}$ in $\mathbb{L}^{S \vee} \oplus \mathbb{Z}$. Then

$$
\begin{aligned}
& \hat{D}_{i}^{S}=\left(D_{i}^{S}, 0\right), \quad \text { for } i \neq j ; \\
& \hat{D}_{j}^{S}=\left(D_{j}^{S},-1\right) ; \quad \hat{D}_{m+l+1}^{S}=\hat{D}_{m+l+2}^{S}=(0,1) ;
\end{aligned}
$$

and

$$
\begin{aligned}
& \hat{D}_{i}=\left(D_{i}, 0\right), \quad \text { for } i \neq j \\
& \hat{D}_{j}=\left(D_{j},-1\right) ; \quad \hat{D}_{m+1}=\hat{D}_{m+2}=(0,1) .
\end{aligned}
$$

The fan $\Sigma_{j}$ of $\mathcal{E}_{j}$ is a rational simplicial fan contained in $\mathbf{N}_{\mathbb{Q}} \oplus \mathbb{Q}$. The 1-skeleton is given by

$$
\begin{aligned}
\hat{b}_{i} & =\left(b_{i}, 0\right), \quad \text { for } 1 \leq i \leq m ; \\
\hat{b}_{m+1} & =(0,1) ; \quad \hat{b}_{m+2}=\left(b_{j},-1\right) .
\end{aligned}
$$

We set

$$
\begin{aligned}
p_{0}^{S} & :=\hat{D}_{m+l+1}^{S}=\hat{D}_{m+l+2}^{S} \in \mathbb{L}^{S \vee} \oplus \mathbb{Z} ; \\
p_{0} & :=\hat{D}_{m+1}=\hat{D}_{m+2} \in H^{2}\left(\mathcal{E}_{j} ; \mathbb{Q}\right) .
\end{aligned}
$$

Then a nef integral basis $\left\{p_{1}, \ldots, p_{r}\right\}$ of $H^{2}(\mathcal{X} ; \mathbb{Q})$ can be lifted to a nef integral basis $\left\{p_{0}, p_{1}, \ldots, p_{r}\right\}$ of $H^{2}\left(\mathcal{E}_{j} ; \mathbb{Q}\right)$ under the splitting (19) (this gives the same splitting as the one in Section 2.1). Recall that $\left\{p_{1}^{S}, \ldots, p_{r+l}^{S}\right\}$ is an integral basis of $\mathbb{L}^{S \vee}$ such that $p_{i}$ is the image of $p_{i}^{S}$ in $\mathbb{L}^{\vee} \otimes \mathbb{R}$. Moreover, $p_{0}^{S}, p_{1}^{S}, \ldots, p_{r+l}^{S}$ is an integral basis of $\mathbb{L}^{S \vee} \oplus \mathbb{Z}$, and $p_{0}$ is the image of $p_{0}^{S}$ in $\left(\mathbb{L}^{\vee} \oplus \mathbb{Z}\right) \otimes \mathbb{R}$. Note that $p_{r+1}, \ldots, p_{r+l}$ are all zero. We have

$$
C_{\mathcal{E}_{j}}^{S}=C_{\mathcal{X}}^{S}+\mathbb{R}_{>0} p_{0}^{S}, \quad \rho_{\mathcal{E}_{j}}^{S}=\rho_{\mathcal{X}}^{S}+p_{0}^{S} .
$$

The following result is straightforward.

Lemma 3.9. If $\rho_{\mathcal{X}}^{S} \in \operatorname{cl}\left(C_{\mathcal{X}}^{S}\right)$, then $\rho_{\mathcal{E}_{j}}^{S} \in \operatorname{cl}\left(C_{\mathcal{E}_{j}}^{S}\right)$ for $1 \leq j \leq m$. 
Definition 3.10. The $I$-function of $\mathcal{X}$ is the $H_{\text {orb }}^{*}(\mathcal{X})$-valued function

$$
\begin{aligned}
I_{\mathcal{X}}(y, z)= & e^{\sum_{i=1}^{r} p_{i} \log y_{i} / z} \\
& \times \sum_{d \in \mathbb{K}_{\mathrm{eff}, \mathcal{X}}} \prod_{i=1}^{m+l}\left(\frac{\prod_{k=\left\lceil\left\langle D_{i}^{S}, d\right\rangle\right\rceil}^{\infty}\left(D_{i}+\left(\left\langle D_{i}^{S}, d\right\rangle-k\right) z\right)}{\prod_{k=0}^{\infty}\left(D_{i}+\left(\left\langle D_{i}^{S}, d\right\rangle-k\right) z\right)}\right) y^{d} \mathbf{1}_{v(d)},
\end{aligned}
$$

where $y^{d}=y_{1}^{\left\langle p_{1}^{S}, d\right\rangle} \cdots y_{r+l}^{\left\langle p_{r+l}^{S}, d\right\rangle}$. Similarly, the $I$-function of $\mathcal{E}$ is the $H_{\text {orb }}^{*}(\mathcal{E})$ valued function

$$
\begin{aligned}
& I_{\mathcal{E}_{j}}(y, z) \\
& \quad=e^{\sum_{i=0}^{r} p_{i} \log y_{i} / z} \\
& \quad \times \sum_{\beta \in \mathbb{K}_{\mathrm{eff}, \mathcal{E}_{j}}} \prod_{i=1}^{m+l+2}\left(\frac{\prod_{k=\left\lceil\left\langle\hat{D}_{i}^{S}, \beta\right\rangle\right\rceil}^{\infty}\left(\hat{D}_{i}+\left(\left\langle\hat{D}_{i}^{S}, \beta\right\rangle-k\right) z\right)}{\prod_{k=0}^{\infty}\left(\hat{D}_{i}+\left(\left\langle\hat{D}_{i}^{S}, \beta\right\rangle-k\right) z\right)}\right) y^{\beta} \mathbf{1}_{v(\beta)},
\end{aligned}
$$

where $y^{\beta}=y_{0}^{\left\langle p_{0}^{S}, \beta,\right\rangle} y_{1}^{\left\langle p_{1}^{S}, \beta\right\rangle} \cdots y_{r+l}^{\left\langle p_{r+l}^{S}, \beta\right\rangle}$.

Following Section 4.1 of [6], the $I$-functions of $\mathcal{X}$ and $\mathcal{E}_{j}$ can be rewritten in the forms

$$
\begin{aligned}
I_{\mathcal{X}}(y, z)= & e^{\sum_{i=1}^{r} p_{i} \log y_{i} / z} \\
& \times \sum_{d \in \mathbb{K}_{\mathcal{X}}} \prod_{i=1}^{m+l}\left(\frac{\prod_{k=\left\lceil\left\langle D_{i}^{S}, d\right\rangle\right\rceil}^{\infty}\left(D_{i}+\left(\left\langle D_{i}^{S}, d\right\rangle-k\right) z\right)}{\prod_{k=0}^{\infty}\left(D_{i}+\left(\left\langle D_{i}^{S}, d\right\rangle-k\right) z\right)}\right) y^{d} \mathbf{1}_{v(d)}
\end{aligned}
$$

and

$$
\begin{aligned}
I_{\mathcal{E}_{j}}(y, z)= & e^{\sum_{i=0}^{r} p_{i} \log y_{i} / z} \\
& \times \sum_{\beta \in \mathbb{K}_{\mathcal{E}_{j}}} \prod_{i=1}^{m+l+2}\left(\frac{\prod_{k=\left\lceil\left\langle\hat{D}_{i}^{S}, \beta\right\rangle\right\rceil}^{\infty}\left(\hat{D}_{i}+\left(\left\langle\hat{D}_{i}^{S}, \beta\right\rangle-k\right) z\right)}{\prod_{k=0}^{\infty}\left(\hat{D}_{i}+\left(\left\langle\hat{D}_{i}^{S}, \beta\right\rangle-k\right) z\right)}\right) y^{\beta} \mathbf{1}_{v(\beta)},
\end{aligned}
$$

respectively, because the summand with $d \in \mathbb{K} \backslash \mathbb{K}_{\mathrm{eff}}$ vanishes. We refer to [6] for more details.

Theorem 3.11 ([4], Thm. 31). Assume that $\rho^{S} \in \operatorname{cl}\left(C_{\mathcal{X}}^{S}\right)$. Then the I-function and the $J$-function satisfy the following relation:

$$
I_{\mathcal{X}}(y, z)=J_{\mathcal{X}}(\tau(y), z),
$$

where

$$
\begin{aligned}
\tau(y) & =\tau_{0,2}(y)+\tau_{t w}(y) \\
& =\sum_{i=1}^{r}\left(\log y_{i}\right) p_{i}+\sum_{j=m+1}^{m+l} y^{D_{j}^{S \vee}} \mathfrak{D}_{j}+\text { h.o.t. } \in H_{\text {orb }}^{\leq 2}(\mathcal{X}),
\end{aligned}
$$

with

$$
\tau_{0,2}(y) \in H^{2}(\mathcal{X}), \quad \tau_{t w}(y) \in H_{t w}^{\leq 2}(\mathcal{X}),
$$




$$
\mathfrak{D}_{j}=\prod_{i \notin I_{j}^{S}} D_{i}^{\left\lfloor c_{j i}\right\rfloor} \mathbf{1}_{v\left(D_{j}^{S \vee}\right)} \in H_{\mathrm{orb}}^{*}(\mathcal{X}),
$$

where h.o.t. (higher-order term) is a fractional power series in $y_{1}, \ldots, y_{r+l}$. Note that under Assumption 3.4, we have $\mathfrak{D}_{j}=1_{s_{j}}$ for $m+1 \leq j \leq m+l$. Furthermore, $\tau(y)$ is called the mirror map and takes values in $H_{\mathrm{orb}}^{\leq 2}(\mathcal{X})$.

Remark 3.12. We only stated a particular case of [4, Thm. 31] when $\mathcal{X}$ is weak Fano. See [6, Sect. 4.1] for more details.

For $\tau_{0,2}(y)=\sum_{a=1}^{r} p_{a} \log q_{a} \in H^{2}(\mathcal{X})$, we have

$$
\log q_{i}=\log y_{i}+g_{i}\left(y_{1}, \ldots, y_{r+l}\right), \quad \text { for } i=1, \ldots, r,
$$

where $g_{i}$ is a (fractional) power series in $y_{1}, \ldots, y_{r+l}$, which is homogeneous of degree zero with respect to the degree $\operatorname{deg} y^{d}=2\left\langle\rho_{\mathcal{X}}^{S}, d\right\rangle$.

By Lemma 3.9, under the assumption of Theorem 3.11, we can also apply the mirror theorem to the associated bundle $\mathcal{E}_{j}$; hence, we have

$$
I_{\mathcal{E}_{j}}(y, z)=J_{\mathcal{E}_{j}}\left(\tau^{(j)}(y), z\right),
$$

where

$$
\tau^{(j)}(y)=\tau_{0,2}^{(j)}+\tau_{t w}^{(j)}(y) \in H^{2}\left(\mathcal{E}_{j}\right) \oplus H_{t w}^{\leq 2}\left(\mathcal{E}_{j}\right)=H_{\mathrm{orb}}^{\leq 2}\left(\mathcal{E}_{j}\right) .
$$

For $\tau_{0,2}^{(j)}(y)=\sum_{a=0}^{r} p_{a} \log q_{a} \in H^{2}\left(\mathcal{E}_{j}\right)$, therefore,

$$
\log q_{i}=\log y_{i}+g_{i}^{(j)}\left(y_{0}, \ldots, y_{r+l}\right) \quad \text { for } i=0, \ldots, r,
$$

where $g_{i}^{(j)}$ is a (fractional) power series in $y_{0}, y_{1}, \ldots, y_{r+l}$, which is homogeneous of degree zero with respect to the degree $\operatorname{deg} y^{\beta}=2\left\langle\rho_{\mathcal{E}_{j}}^{S}, \beta\right\rangle$.

\subsection{Seidel Elements and Mirror Maps}

Proposition 3.13. The function $g_{i}^{(j)}$ does not depend on $y_{0}$, and we have

$$
g_{i}^{(j)}\left(y_{0}, \ldots, y_{r+l}\right)=g_{i}\left(y_{1}, \ldots, y_{r+l}\right) \text { for } i=1, \ldots, r .
$$

Proof. The functions $g_{i}$ are the coefficients of $z^{-1} p_{i}$ in the expansion of $I_{\mathcal{X}}$ :

$$
I_{\mathcal{X}}(y, z)=e^{\sum_{i=1}^{r} p_{i} \log y_{i} / z}\left(1+z^{-1}\left(\sum_{i=1}^{r} g_{i}(y) p_{i}+\tau_{t w}\right)+O\left(z^{-2}\right)\right) .
$$

The functions $g_{i}^{(j)}$ are the coefficients of $z^{-1} p_{i}$ in the expansion of $I_{\mathcal{E}_{j}}$ :

$$
I_{\mathcal{E}_{j}}(y, z)=e^{\sum_{i=0}^{r} p_{i} \log y_{i} / z}\left(1+z^{-1}\left(\sum_{i=0}^{r} g_{i}^{(j)}(y) p_{i}+\tau_{t w}^{(j)}\right)+O\left(z^{-2}\right)\right) .
$$

Following the proof of Lemma 3.5 of [5], we obtain the conclusion of this proposition. 
We will prove that $\tau_{t w}^{(j)}(y)$ is also independent from $y_{0}$. To begin with, the following lemma implies that $\tau_{t w}^{(j)}(y)$ is an (integer) power series in $y_{0}$.

Lemma 3.14. For any $\beta \in \mathbb{K}_{\mathcal{E}_{j}}$, we have $\left\langle p_{0}^{S}, \beta\right\rangle \in \mathbb{Z}$. Furthermore, for any $\beta \in \mathbb{K}_{\mathrm{eff}, \mathcal{E}_{j}}$, we have $\left\langle p_{0}^{S}, \beta\right\rangle \in \mathbb{Z}_{\geq 0}$.

Proof. Any cone $\sigma \in \Sigma_{j}$ containing both $\hat{b}_{m+1}$ and $\hat{b}_{m+2}$ should also contain $\hat{b}_{j}$, but this is impossible since the fan $\Sigma_{j}$ is simplicial and $\hat{b}_{m+1}, \hat{b}_{m+2}$, and $\hat{b}_{j}$ lie in the same plane. Hence, by the definition of $\mathbb{K}_{\mathcal{E}_{j}}$ (resp. $\mathbb{K}_{\mathrm{eff}, \mathcal{E}_{j}}$ ) at least one of $\left\langle\hat{D}_{m+1}^{S}, \beta\right\rangle$ and $\left\langle\hat{D}_{m+2}^{S}, \beta\right\rangle$ has to be integer (resp. nonnegative integer) for any $\beta \in \mathbb{K}_{\mathcal{E}_{j}}$ (resp. $\beta \in \mathbb{K}_{\mathrm{eff}, \mathcal{E}_{j}}$ ). On the other hand, we have

$$
\left\langle p_{0}^{S}, \beta\right\rangle=\left\langle\hat{D}_{m+1}^{S}, \beta\right\rangle=\left\langle\hat{D}_{m+2}^{S}, \beta\right\rangle .
$$

Therefore, we must have $\left\langle p_{0}^{S}, \beta\right\rangle \in \mathbb{Z}$ (resp. $\left\langle p_{0}^{S}, \beta\right\rangle \in \mathbb{Z}_{\geq 0}$ ).

As a direct consequence of the lemma, $\tau_{t w}^{(j)}(y)$ can only contain nonnegative integer powers of $y_{0}$.

Proposition 3.15. Let $\tau_{t w}^{(j)}(y)=\sum_{n=0}^{\infty} P_{n}^{(j)}(y) y_{0}^{n}$, where $P_{n}^{(j)}(y)$ is a fractional) power series in $y_{1}, \ldots, y_{n}$. Then

$$
P_{n}^{(j)}(y)=0 \quad \text { for } n \geq 1,
$$

that is, $\tau_{t w}^{(j)}(y)$ is independent from $y_{0}$. Moreover, we have

$$
\imath^{*} \tau_{t w}^{(j)}(y)=\tau_{t w}(y)
$$

Proof. Recall that $\tau_{t w}^{(j)}(y)$ is the coefficient of $z^{-1}$ in

$$
\begin{aligned}
e^{-\sum_{i=0}^{r} p_{i} \log y_{i} / z} I_{\mathcal{E}_{j}}(y, z) & \\
= & \sum_{\beta \in \mathbb{K}_{\mathrm{eff}, \mathcal{E}_{j}}} \prod_{i=1}^{m+l+2}\left(\frac{\prod_{k=\left\lceil\left\langle\hat{D}_{i}^{S}, \beta\right\rangle\right\rceil}^{\infty}\left(\hat{D}_{i}+\left(\left\langle\hat{D}_{i}^{S}, \beta\right\rangle-k\right) z\right)}{\prod_{k=0}^{\infty}\left(\hat{D}_{i}+\left(\left\langle\hat{D}_{i}^{S}, \beta\right\rangle-k\right) z\right)}\right) y^{\beta} \mathbf{1}_{v(\beta)},
\end{aligned}
$$

valued in $H_{t w}^{\leq 2}\left(\mathcal{E}_{j}\right)$. Hence, we only need to consider terms with $v(\beta) \neq 0$. On the other hand, by Lemma 3.14 we have

$$
\left\langle p_{0}^{S}, \beta\right\rangle=\left\langle\hat{D}_{m+1}^{S}, \beta\right\rangle=\left\langle\hat{D}_{m+2}^{S}, \beta\right\rangle \in \mathbb{Z} \quad \text { for } \beta \in \mathbb{K}_{\mathcal{E}_{j}} .
$$

Hence, we obtain

$$
v(\beta)=\sum_{i=1}^{m+2}\left\{-\left\langle\hat{D}_{i}^{S}, \beta\right\rangle\right\} \hat{b}_{i}=\left(\sum_{i=1}^{m}\left\{-\left\langle D_{i}^{S}, d\right\rangle\right\} b_{i}, 0\right)=(v(d), 0) \in \mathbf{N} \oplus \mathbb{Z},
$$

where $d$ is the natural projection of $\beta$ onto $\mathbb{K}_{\mathrm{eff}, \mathcal{X}}$. Therefore, $v(\beta) \neq 0$ is equivalent to $v(d) \neq 0$. 
It remains to examine the product factor:

$$
\begin{aligned}
& \prod_{i=1}^{m+l+2}\left(\frac{\prod_{k=\left\lceil\left\langle\hat{D}_{i}^{S}, \beta\right\rangle\right\rceil}^{\infty}\left(\hat{D}_{i}+\left(\left\langle\hat{D}_{i}^{S}, \beta\right\rangle-k\right) z\right)}{\prod_{k=0}^{\infty}\left(\hat{D}_{i}+\left(\left\langle\hat{D}_{i}^{S}, \beta\right\rangle-k\right) z\right)}\right) \\
& =\frac{\prod_{i:\left\langle\hat{D}_{i}^{S}, \beta\right\rangle<0} \prod_{\left\langle\hat{D}_{i}^{S}, \beta\right\rangle \leq k<0}\left(\hat{D}_{i}+\left(\left\langle\hat{D}_{i}^{S}, \beta\right\rangle-k\right) z\right)}{\prod_{i:\left\langle\hat{D}_{i}^{S}, \beta\right\rangle>0} \prod_{0 \leq k<\left\langle\hat{D}_{i}^{S}, \beta\right\rangle}\left(\hat{D}_{i}+\left(\left\langle\hat{D}_{i}^{S}, \beta\right\rangle-k\right) z\right)} \\
& =C_{\beta} z^{-\left(\sum_{i=1}^{m+l+2}\left\lceil\left\langle\hat{D}_{i}^{S}, \beta\right\rangle\right\rceil+\#\left\{i:\left\langle\hat{D}_{i}^{S}, \beta\right\rangle \in \mathbb{Z}_{<0}\right\}\right)} \prod_{i:\left\langle\hat{D}_{i}^{S}, \beta\right\rangle \in \mathbb{Z}_{<0}} \hat{D}_{i}+\text { h.o.t. }
\end{aligned}
$$

where

$$
\begin{aligned}
C_{\beta}= & \prod_{i:\left\langle\hat{D}_{i}^{S}, \beta\right\rangle<0} \prod_{\left\langle\hat{D}_{i}^{S}, \beta\right\rangle<k<0}\left(\left\langle\hat{D}_{i}^{S}, \beta\right\rangle-k\right) \\
& \times \prod_{i:\left\langle\hat{D}_{i}^{S}, \beta\right\rangle>0} \prod_{0 \leq k<\left\langle\hat{D}_{i}^{S}, \beta\right\rangle}\left(\left\langle\hat{D}_{i}^{S}, \beta\right\rangle-k\right)^{-1} .
\end{aligned}
$$

By assumption we need to have

$$
\sum_{i=1}^{m+l+2}\left\lceil\left\langle\hat{D}_{i}^{S}, \beta\right\rangle\right\rceil \geq \sum_{i=1}^{m+l+2}\left\langle\hat{D}_{i}^{S}, \beta\right\rangle \geq 0 .
$$

The equality holds if and only if

$$
\left\langle\hat{D}_{i}^{S}, \beta\right\rangle \in \mathbb{Z} \quad \text { for all } 1 \leq i \leq m+l+2 \quad \text { and } \quad \sum_{i=1}^{m+l+2}\left\langle\hat{D}_{i}^{S}, \beta\right\rangle=0 .
$$

However, this would imply $v(\beta)=0$, and hence we cannot have $\sum_{i=1}^{m+l+2}\left\lceil\left\langle\hat{D}_{i}^{S}, \beta\right\rangle\right\rceil=0$. Therefore, expansion (30) would contribute to $P_{n}^{(j)}$ only when

$$
\sum_{i=1}^{m+l+2}\left\lceil\left\langle\hat{D}_{i}^{S}, \beta\right\rangle\right\rceil=1 \quad \text { and } \quad \#\left\{i:\left\langle\hat{D}_{i}^{S}, \beta\right\rangle \in \mathbb{Z}_{<0}\right\}=0 .
$$

In this case, if $\left\langle p_{0}^{S}, \beta\right\rangle \geq 1$, then

$$
\sum_{i=1}^{m+l+2}\left\lceil\left\langle\hat{D}_{i}^{S}, \beta\right\rangle\right\rceil \geq \sum_{i=1}^{m+l}\left\lceil\left\langle D_{i}^{S}, d\right\rangle\right\rceil+1
$$

and, therefore, we have

$$
0 \geq \sum_{i=1}^{m+l}\left\lceil\left\langle D_{i}^{S}, d\right\rangle\right\rceil \geq \sum_{i=1}^{m+l}\left\langle D_{i}^{S}, d\right\rangle=0 .
$$

This implies that when $\left\langle p_{0}^{S}, \beta\right\rangle \geq 1$, we must have

$$
\left\langle D_{i}^{S}, d\right\rangle \in \mathbb{Z} \quad \text { for } 1 \leq i \leq m+l .
$$


It is a contradiction since $\tau_{t w}^{(j)}(y) \in H_{t w}^{\leq 2}\left(\mathcal{E}_{j}\right)$ implies $v(\beta) \neq 0$, equivalently, $v(d) \neq 0$. Hence,

$$
P_{n}^{(j)}=0 \text { for all } n>0,
$$

and $\tau_{t w}^{(j)}(y)$ is independent from $y_{0}$. Moreover, by the expression of $I$-functions and the identity

$$
\left.\iota^{*} I_{\mathcal{E}_{j}}\right|_{y_{0}=0}=I \mathcal{X}
$$

we have $\iota^{*} \tau_{t w}^{(j)}(y)=\tau_{t w}(y)$.

Since $\tilde{S}_{j}\left(\tau^{(j)}(y)\right)$ does not depend on $y_{0}$ or $q_{0}$, we use the following notation for the Seidel element:

$$
\tilde{S}_{j}(\tau(y)):=\tilde{S}_{j}\left(\tau^{(j)}(y)\right) \text {. }
$$

\subsection{Seidel Elements in Terms of I-Functions}

We can rewrite the $I$-function of the associated bundle $\mathcal{E}_{j}$ as follows:

$$
\begin{gathered}
e^{\sum_{i=0}^{r} p_{i} \log y_{i} / z}\left(1+z^{-1}\left(\sum_{i=0}^{r} g_{i}^{(j)}(y) p_{i}+\tau_{t w}^{(j)}(y)\right)\right. \\
\left.+z^{-2}\left(\sum_{n=0}^{2} G_{n}^{(j)}(y) y_{0}^{n}\right)+O\left(z^{-3}\right)\right) .
\end{gathered}
$$

Then, $\log q_{i}=\log y_{i}+g_{i}^{(j)}(y)$ implies

$$
\begin{aligned}
I_{\mathcal{E}_{j}}(y, z)= & e^{\sum_{i=0}^{r} p_{i} \log q_{i} / z}\left(1+z^{-1} \tau_{t w}^{(j)}(y)\right. \\
& \left.+z^{-2}\left(\sum_{n=0}^{2} G_{n}^{(j)}(y) y_{0}^{n}\right)+O\left(z^{-3}\right)\right),
\end{aligned}
$$

where $G_{n}^{(j)}(y)$ is a (fractional) power series in $y_{1}, \ldots, y_{r+l}$ taking values in $H_{\text {orb }}^{*}\left(\mathcal{E}_{j}\right)$. in

By Proposition 2.6 the Seidel element $\tilde{S}_{j}\left(\tau^{(j)}(y)\right)$ is the coefficient of $q_{0} / z^{2}$

$$
\exp \left(-\sum_{i=0}^{r} p_{i} \log q_{i} / z\right) J_{\mathcal{E}_{j}}\left(\tau^{(j)}(y), z\right)
$$

hence, $J_{\mathcal{E}_{j}}\left(\tau^{(j)}(y), z\right)=I_{\mathcal{E}_{j}}(y, z)$ and $\log q_{0}=\log y_{0}+g_{0}^{(j)}(y)$ imply the following result.

THeOREM 3.16. The Seidel element $S_{j}$ associated to the toric divisor $D_{j}$ is given by

$$
S_{j}\left(\tau^{(j)}(y)\right)=\iota^{*}\left(G_{1}^{(j)}(y) y_{0}\right)
$$

Furthermore, we have

$$
\tilde{S}_{j}(\tau(y))=\tilde{S}_{j}\left(\tau^{(j)}(y)\right)=\exp \left(-g_{0}^{j}(y)\right) \iota^{*}\left(G_{1}^{(j)}(y)\right) .
$$




\subsection{Computation of $g_{0}^{(j)}$}

The computation is essentially the same as the proof of Lemma 3.16 of [5]. Consider the product factors in $I_{\mathcal{E}_{j}}$ :

$$
\prod_{i=1}^{m+l+2}\left(\frac{\prod_{k=\left\lceil\left\langle\hat{D}_{i}^{S}, \beta\right\rangle\right\rceil}^{\infty}\left(\hat{D}_{i}+\left(\left\langle\hat{D}_{i}^{S}, \beta\right\rangle-k\right) z\right)}{\prod_{k=0}^{\infty}\left(\hat{D}_{i}+\left(\left\langle\hat{D}_{i}^{S}, \beta\right\rangle-k\right) z\right)}\right) y^{\beta} \mathbf{1}_{v(\beta)} .
$$

These factors contribute to $g_{i}^{(j)}$ if

$$
v(\beta)=\sum_{i=1}^{m+l+2}\left\{-\left\langle\hat{D}_{i}^{S}, \beta\right\rangle\right\} \hat{b}_{i}=0 .
$$

Then, by the definition of $\mathbb{K}_{\text {eff }}$ we must have

$$
\left\langle\hat{D}_{i}^{S}, \beta\right\rangle \in \mathbb{Z} \quad \text { for all } 1 \leq i \leq m+l+2 .
$$

In this case, the product factors can be rewritten as

$$
\begin{aligned}
& \prod_{i=1}^{m+l+2}\left(\frac{\prod_{k=\left\lceil\left\langle\hat{D}_{i}^{S}, \beta\right\rangle\right\rceil}^{\infty}\left(\hat{D}_{i}+\left(\left\langle\hat{D}_{i}^{S}, \beta\right\rangle-k\right) z\right)}{\prod_{k=0}^{\infty}\left(\hat{D}_{i}+\left(\left\langle\hat{D}_{i}^{S}, \beta\right\rangle-k\right) z\right)}\right) y^{\beta} \mathbf{1}_{v(\beta)} \\
& \quad=\prod_{i=1}^{m+l+2} \frac{\prod_{k=-\infty}^{0}\left(\hat{D}_{i}+k z\right)}{\prod_{k=-\infty}^{\left\langle\hat{D}_{i}^{S}, \beta\right\rangle}\left(\hat{D}_{i}+k z\right)} y^{\beta} \\
& \quad=\left(C_{\beta} z^{-\sum_{i=1}^{m+l+2}\left\langle\hat{D}_{i}^{S}, \beta\right\rangle-\#\left\{i:\left\langle\hat{D}_{i}^{S}, \beta\right\rangle<0\right\}} \prod_{i:\left\langle\hat{D}_{i}^{S}, \beta\right\rangle<0} \hat{D}_{i}+\text { h.o.t. }\right) y^{\beta}
\end{aligned}
$$

where h.o.t. stands for higher-order terms in $z^{-1}$, and

$$
C_{\beta}=\prod_{i:\left\langle\hat{D}_{i}^{S}, \beta\right\rangle<0}(-1)^{-\left\langle\hat{D}_{i}^{S}, \beta\right\rangle-1}\left(-\left\langle\hat{D}_{i}^{S}, \beta\right\rangle-1\right) ! \prod_{i:\left\langle\hat{D}_{i}^{S}, \beta\right\rangle \geq 0}\left(\left\langle\hat{D}_{i}^{S}, \beta\right\rangle !\right)^{-1} .
$$

They contribute to the $z^{-1}$ term if

$$
\sum_{i=1}^{m+l+2}\left\langle\hat{D}_{i}^{S}, \beta\right\rangle+\#\left\{i:\left\langle\hat{D}_{i}^{S}, \beta\right\rangle<0\right\} \leq 1 .
$$

Since by assumption $\rho_{\mathcal{X}}^{S} \in \operatorname{cl}\left(C_{\mathcal{X}}^{S}\right)$, we have $\rho_{\mathcal{E}_{j}}^{S} \in \operatorname{cl}\left(C_{\mathcal{E}_{j}}^{S}\right)$. So there are the following three cases:

- $\left\{\begin{array}{l}\sum_{i=1}^{m+l+2}\left\langle\hat{D}_{i}^{S}, \beta\right\rangle=0, \\ \#\left\{i:\left\langle\hat{D}_{i}^{S}, \beta\right\rangle \in \mathbb{Z}_{<0}\right\}=0 ;\end{array}\right.$

- $\left\{\begin{array}{l}\sum_{i=1}^{m+l+2}\left\langle\hat{D}_{i}^{S}, \beta\right\rangle=1, \\ \#\left\{i:\left\langle\hat{D}_{i}^{S}, \beta\right\rangle \in \mathbb{Z}_{<0}\right\}=0 ;\end{array}\right.$

- $\left\{\begin{array}{l}\left.\sum_{i=1}^{m+l+2}\left\langle\hat{D}_{i}^{S}, \beta\right\rangle\right\rceil=0, \\ \#\left\{i:\left\langle\hat{D}_{i}^{S}, \beta\right\rangle \in \mathbb{Z}_{<0}\right\}=1 .\end{array}\right.$ 
In the first case, we have $\left\langle\hat{D}_{i}^{S}, \beta\right\rangle=0$ for all $i$, and hence $\beta=0$; the second case cannot happen, since $\beta$ has to satisfy $\left\langle\hat{D}_{i}^{S}, \beta\right\rangle=0$ except for one $i$, and this implies $\beta=0$. Therefore, the coefficient of $z^{-1}$ is from the third case, where

$$
\sum_{i=1}^{m+l+2}\left\langle\hat{D}_{i}^{S}, \beta\right\rangle=0 \quad \text { and } \quad \#\left\{i:\left\langle\hat{D}_{i}^{S}, \beta\right\rangle<0\right\}=1 .
$$

By the assumption $\rho_{\mathcal{X}}^{S} \in \operatorname{cl}\left(C_{\mathcal{X}}^{S}\right)$ we must have $\sum_{i=1}^{m+l}\left\langle D_{i}^{S}, d\right\rangle=0$ and $\left\langle p_{0}^{S}, \beta\right\rangle=0$. Moreover, $\left\langle D_{i}^{S}, d\right\rangle<0$ for exactly one $i$ in $\{1, \ldots, m\}$. (Note that $\left\langle D_{i}^{S}, d\right\rangle \geq 0$ for $i \in\{m+1, \ldots, m+l\}$.)

Now $g_{0}^{(j)}$ is the coefficient corresponding to $p_{0}$, and $\hat{D}_{j}=\left\langle D_{j},-1\right\rangle=$ $D_{j}-p_{0}$ is the only one, among $\hat{D}_{1}, \ldots, \hat{D}_{m}$, which contains $p_{0}$. By expression (37) we must have $\left\langle D_{j}^{S}, d\right\rangle<0$ and $\left\langle D_{i}^{S}, d\right\rangle \geq 0$ for $i \neq j$. Hence, we have

LEMma 3.17. The coefficient $g_{0}^{(j)}$ is given by

$$
g_{0}^{j}\left(y_{1}, \ldots, y_{r+l}\right)=\sum_{\substack{\left\langle D_{i}^{S}, d\right\rangle \in \mathbb{Z}, 1 \leq i \leq m+l \\\left\langle\rho_{\mathcal{X}}^{S}, d\right\rangle=0 \\\left\langle D_{j}^{S}, d\right\rangle<0 \\\left\langle D_{i}^{S}, d\right\rangle \geq 0, \forall i \neq j}} \frac{(-1)^{-\left\langle D_{j}^{S}, d\right\rangle}\left(-\left\langle D_{j}^{S}, d\right\rangle-1\right) !}{\prod_{i \neq j}\left\langle D_{i}^{S}, d\right\rangle !} y^{d} .
$$

\section{Batyrev Elements}

In this section, we extend the definition of Batyrev elements in [5] to toric Deligne-Mumford stacks and explore their relationships with Seidel elements. Batyrev elements satisfy the multiplicative and linear Batyrev relations as in Batyrev's presentation of quantum cohomology ring for toric manifolds in [1].

\subsection{Batyrev Elements}

Following [6], consider the mirror coordinates $y_{1}, \ldots, y_{r+l}$ of the toric DeligneMumford stacks $\mathcal{X}$ with $\rho_{\mathcal{X}}^{S} \in \operatorname{cl}\left(C_{\mathcal{X}}^{S}\right)$. Set $\mathbb{C}\left[y^{ \pm}\right]=\mathbb{C}\left[y_{1}^{ \pm}, \ldots, y_{r+l}^{ \pm}\right]$.

Definition 4.1 ([6]). The Batyrev ring $B(\mathcal{X})$ of $\mathcal{X}$ is a $\mathbb{C}\left[y^{ \pm}\right]$-algebra generated by the variables $\lambda_{1}, \ldots, \lambda_{r+l}$ with the following two Batyrev relations:

$$
\begin{gathered}
\text { (multiplicative): } y^{d} \prod_{i:\left\langle D_{i}^{S}, d\right\rangle<0} \omega_{i}^{-\left\langle D_{i}^{S}, d\right\rangle}=\prod_{i:\left\langle D_{i}^{S}, d\right\rangle>0} \omega_{i}^{\left\langle D_{i}^{S}, d\right\rangle}, d \in \mathbb{L}^{S} ; \\
\text { (linear): } \omega_{i}=\sum_{a=1}^{r+l} m_{a i} \lambda_{a},
\end{gathered}
$$

where $\omega_{i}$ is invertible in $B(\mathcal{X})$. 
Definition 4.2. Let $\mathbb{Q}\left\langle\left\langle y_{1}, \ldots, y_{r+l}\right\rangle\right\rangle$ be the field of fractional power series of $y_{1}, \ldots, y_{r+l}$. We define the element $\tilde{p}_{i}^{S} \in H_{\text {orb }}^{\leq 2}(\mathcal{X}) \otimes \mathbb{Q}\left\langle\left\langle y_{1}, \ldots, y_{r+l}\right\rangle\right\rangle$ as

$$
\tilde{p}_{i}^{S}=\frac{\partial \tau(y)}{\partial \log y_{i}}, \quad i=1, \ldots, r+l .
$$

Recall that

$$
D_{j}^{S}=\sum_{i=1}^{r+l} m_{i j} p_{i}^{S}, \quad \text { for } 1 \leq j \leq m+l,
$$

Then, the Batyrev element associated to $D_{j}^{S}$ is defined by

$$
\tilde{D}_{j}^{S}=\sum_{i=1}^{r+l} m_{i j} \tilde{p}_{i}^{S} \quad \text { for } 1 \leq j \leq m+l .
$$

Proposition 4.3. The Batyrev elements $\tilde{D}_{1}^{S}, \ldots, \tilde{D}_{m+l}^{S}$ satisfy the multiplicative and linear Batyrev relations for $\omega_{j}=\tilde{D}_{j}^{S}$.

Proof. We consider the differential operator $\mathcal{P}_{d} \in \mathbb{C}\left[z, y^{ \pm}, z y(\partial / \partial y)\right]$ for $d \in \mathbb{L}^{S}$, introduced by Iritani in [6], Section 4.2:

$$
\mathcal{P}_{d}:=y^{d} \prod_{i:\left\langle D_{i}^{S}, d\right\rangle<0} \prod_{k=0}^{-\left\langle D_{i}^{S}, d\right\rangle-1}\left(\mathcal{D}_{i}-k z\right)-\prod_{i:\left\langle D_{i}^{S}, d\right\rangle>0} \prod_{k=0}^{\left\langle D_{i}^{S}, d\right\rangle-1}\left(\mathcal{D}_{i}-k z\right),
$$

where $\mathcal{D}_{i}:=\sum_{j=1}^{r+l} m_{i j} z y_{j} \partial / \partial y_{j}$.

By [6, Lemma 4.6], we have

$$
\mathcal{P}_{d} I(y, z)=0, \quad d \in \mathbb{L}^{S} .
$$

Hence,

$$
0=\mathcal{P}_{d}(z, y, z y \partial / \partial y) I(y, z)=\mathcal{P}_{d}(z, y, z y \partial / \partial y) J(\tau(y), z) .
$$

This implies that

$$
\mathcal{P}_{d}\left(z, y, z \tau^{*} \nabla\right) \mathbf{1}=0,
$$

where $\tau^{*} \nabla_{i}:=\nabla_{\tau_{*}\left(y_{i}\left(\partial / \partial y_{i}\right)\right)}$. Since

$$
\tau(y)=\sum_{i=1}^{r} p_{i} \log y_{i}+\tau_{t w}(y)
$$

and

$$
\nabla_{\tau_{*}\left(y_{i}\left(\partial / \partial y_{i}\right)\right)}=\tau_{*}\left(y_{i}\left(\partial / \partial y_{i}\right)\right)+\frac{1}{z} y_{i} \frac{\partial \tau(y)}{\partial y_{i}} \circ_{\tau},
$$

by setting $z=0$ we proved that the Batyrev elements satisfy the multiplicative relation.

It is straightforward from the definition that the Batyrev elements satisfy the linear relation. 
Consider the $I$-function for the bundle $\mathcal{E}_{j}$ associated to the toric divisor $D_{j}^{S}$ for $1 \leq j \leq m$ :

$$
\begin{aligned}
I_{\mathcal{E}_{j}}(y, z) & =e^{\sum_{i=0}^{r} p_{i} \log y_{i} / z} \\
& \times \sum_{\beta \in \mathbb{K}_{\mathcal{E}_{j}}} \prod_{i=1}^{m+l+2}\left(\frac{\prod_{k=\left\lceil\left\langle\hat{D}_{i}^{S}, \beta\right\rangle\right\rceil}^{\infty}\left(\hat{D}_{i}+\left(\left\langle\hat{D}_{i}^{S}, \beta\right\rangle-k\right) z\right)}{\prod_{k=0}^{\infty}\left(\hat{D}_{i}+\left(\left\langle\hat{D}_{i}^{S}, \beta\right\rangle-k\right) z\right)}\right) y^{\beta} \mathbf{1}_{v(\beta)},
\end{aligned}
$$

where $y^{\beta}=y_{0}^{\left\langle p_{0}^{S}, \beta,\right\rangle} y_{1}^{\left\langle p_{1}^{S}, \beta\right\rangle} \cdots y_{r+l}^{\left\langle p_{r+l}^{S}, \beta\right\rangle}$. The following lemma is a generalization of Lemma 3.11 in [5].

LEMma 4.4. The I-function $I_{\mathcal{E}_{j}}$ of the bundle $\mathcal{E}_{j}$, associated to the toric divisor $D_{j}^{S}$, satisfies the following partial differential equation:

$$
z \frac{\partial}{\partial y_{0}}\left(y_{0} \frac{\partial}{\partial y_{0}}\right) I_{\mathcal{E}_{j}}=\left(\sum_{i=1}^{r+l} m_{i j}\left(y_{i} \frac{\partial}{\partial y_{i}}\right)-y_{0} \frac{\partial}{\partial y_{0}}\right) I_{\mathcal{E}_{j}} .
$$

Proof. It follows from applying the operator $\mathcal{P}_{\beta}$ defined in equation (42) to the bundle $\mathcal{E}_{j}$ with $\beta=\left[\sigma_{0}\right]$.

Using the expansion of $I_{\mathcal{E}_{j}}$, we have

$$
\begin{aligned}
I_{\mathcal{E}_{j}}(y, z)= & e^{\sum_{i=0}^{r} p_{i} \log y_{i} / z}\left(1+z^{-1}\left(\sum_{i=0}^{r} g_{i}^{(j)}(y) p_{i}+\tau_{t w}^{(j)}\right)\right. \\
& \left.+z^{-2}\left(\sum_{n=0}^{2} G_{n}^{(j)}(y) y_{0}^{n}\right)+O\left(z^{-3}\right)\right),
\end{aligned}
$$

where $G_{n}^{(j)}$ is a (fractional) power series in $y_{1}, \ldots, y_{r+l}$ taking values in $H_{\text {orb }}^{*}\left(\mathcal{E}_{j}\right)$. Therefore, we obtain

$$
\begin{aligned}
y_{0} \frac{\partial}{\partial y_{0}} I_{\mathcal{E}_{j}}= & \frac{p_{0}}{z} e^{\sum_{i=0}^{r} p_{i} \log y_{i} / z}\left(1+z^{-1}\left(\sum_{i=0}^{r} g_{i}^{(j)}(y) p_{i}+\tau_{t w}^{(j)}\right)\right. \\
& \left.+z^{-2}\left(\sum_{n=0}^{2} G_{n}^{(j)}(y) y_{0}^{n}\right)+O\left(z^{-3}\right)\right) \\
& +e^{\sum_{i=0}^{r} p_{i} \log y_{i} / z}\left(z^{-2}\left(\sum_{n=1}^{2} G_{n}^{(j)}(y) n y_{0}^{n}\right)+O\left(z^{-3}\right)\right) .
\end{aligned}
$$

Therefore, the left-hand side of equation (43) is

$$
\begin{aligned}
& z \frac{\partial}{\partial y_{0}}\left(y_{0} \frac{\partial}{\partial y_{0}}\right) I_{\mathcal{E}_{j}} \\
& \quad=\frac{\partial}{\partial y_{0}}\left(p _ { 0 } e ^ { \sum _ { i = 0 } ^ { r } p _ { i } \operatorname { l o g } y _ { i } / z } \left(1+z^{-1}\left(\sum_{i=0}^{r} g_{i}^{(j)}(y) p_{i}+\tau_{t w}^{(j)}\right)\right.\right.
\end{aligned}
$$




$$
\begin{aligned}
& \left.\left.+z^{-2}\left(\sum_{n=0}^{2} G_{n}^{(j)}(y) y_{0}^{n}\right)+O\left(z^{-3}\right)\right)\right) \\
& +\frac{\partial}{\partial y_{0}}\left(e^{\sum_{i=0}^{r} p_{i} \log y_{i} / z}\left(z^{-1}\left(\sum_{n=1}^{2} G_{n}^{(j)}(y) n y_{0}^{n}\right)+O\left(z^{-2}\right)\right)\right) \\
& =p_{0} e^{\sum_{i=0}^{r} p_{i} \log y_{i} / z}\left(O\left(z^{-2}\right)\right) \\
& +\frac{p_{0}}{y_{0} z} e^{\sum_{i=0}^{r} p_{i} \log y_{i} / z}\left(z^{-1}\left(\sum_{n=1}^{2} G_{n}^{(j)}(y) n y_{0}^{n}\right)+O\left(z^{-2}\right)\right) \\
& +e^{\sum_{i=0}^{r} p_{i} \log y_{i} / z}\left(z^{-1}\left(\sum_{n=1}^{2} G_{n}^{(j)} n^{2} y_{0}^{n-1}+O\left(z^{-2}\right)\right)\right) \\
& =e^{\sum_{i=0}^{r} p_{i} \log y_{i} / z}\left(z^{-1}\left(\sum_{n=1}^{2} G_{n}^{(j)} n^{2} y_{0}^{n-1}\right)+O\left(z^{-2}\right)\right) .
\end{aligned}
$$

On the other hand, the pull-back of the right-hand side of equation (43) by $\imath^{*}$ is

$$
\begin{aligned}
& \iota^{*}\left(\sum_{i=1}^{r+l} m_{i j}\left(y_{i} \frac{\partial}{\partial y_{i}}\right)-y_{0} \frac{\partial}{\partial y_{0}}\right) I_{\mathcal{E}_{j}} \\
& \quad=\left(\sum_{i=1}^{r+l} m_{i j}\left(y_{i} \frac{\partial}{\partial y_{i}}\right)-y_{0} \frac{\partial}{\partial y_{0}}\right) \iota^{*} I_{\mathcal{E}_{j}} \\
& \quad=\left(\sum_{i=1}^{r+l} m_{i j}\left(y_{i} \frac{\partial}{\partial y_{i}}\right)\right)\left(I \mathcal{X}+O\left(y_{0}\right)\right) \\
& \quad=z^{-1}\left(\sum_{i=1}^{r+l} m_{i j}\left(y_{i} \frac{\partial}{\partial y_{i}}\right) \tau(y)\right)+O\left(z^{-2}\right)+O\left(y_{0}\right) .
\end{aligned}
$$

Hence, we conclude the following lemma.

Lemma 4.5. The Batyrev element $\tilde{D}_{j}(y)$ is given by

$$
\tilde{D}_{j}(y)=l^{*} G_{1}^{(j)}(y) \quad \text { for } 1 \leq j \leq m+l .
$$

Hence, the following theorem is a direct consequence of the last lemma and Theorem 3.16.

TheOREM 4.6. The Seidel element $\tilde{S}_{j}$ corresponding to the toric divisor $D_{j}$ is given by

$$
\tilde{S}_{j}(\tau(y))=\exp \left(-g_{0}^{j}(y)\right) \tilde{D}_{j}(y) .
$$




\subsection{The Computation of $\tilde{D}_{j}$}

Using the expansion

$$
\left(\sum_{i=1}^{r+l} m_{i j}\left(y_{i} \frac{\partial}{\partial y_{i}}\right)\right) I \mathcal{X}=e^{\sum_{i=1}^{r} p_{i} \log y_{i} / z}\left(z^{-1} \tilde{D}_{j}+O\left(z^{-2}\right)\right),
$$

we see that $\tilde{D}_{j}$ is the coefficient of $z^{-1}$ in the expansion of

$$
e^{-\sum_{i=1}^{r} p_{i} \log y_{i} / z}\left(\sum_{i=1}^{r+l} m_{i j}\left(y_{i} \frac{\partial}{\partial y_{i}}\right)\right) I_{\mathcal{X}} .
$$

By direct computation we get

$$
\begin{aligned}
\left(\sum_{i=1}^{r+l} m_{i j}\left(y_{i} \frac{\partial}{\partial y_{i}}\right)\right) I \mathcal{X} & \\
= & e^{\sum_{i=1}^{r} p_{i} \log y_{i} / z} \sum_{d \in \mathbb{K}_{\mathrm{eff}, \mathcal{X}}} \prod_{i=1}^{m+l}\left(\frac{\prod_{k=\left\lceil\left\langle D_{i}^{S}, d\right\rangle\right\rceil}^{\infty}\left(D_{i}+\left(\left\langle D_{i}^{S}, d\right\rangle-k\right) z\right)}{\prod_{k=0}^{\infty}\left(D_{i}+\left(\left\langle D_{i}^{S}, d\right\rangle-k\right) z\right)}\right) \\
& \times\left(\frac{D_{j}}{z}+\left\langle D_{j}^{S}, d\right\rangle\right) y^{d} \mathbf{1}_{v(d)} .
\end{aligned}
$$

Hence, to compute the Batyrev element $\tilde{D}_{j}$, it remains to examine the expansion of the product factor

$$
\begin{aligned}
& \frac{\prod_{k=\left\lceil\left\langle D_{i}^{S}, d\right\rangle\right\rceil}^{\infty}\left(D_{i}+\left(\left\langle D_{i}^{S}, d\right\rangle-k\right) z\right)}{\prod_{k=0}^{\infty}\left(D_{i}+\left(\left\langle D_{i}^{S}, d\right\rangle-k\right) z\right)} \\
& \quad=C_{d} z^{-\left(\sum_{i=1}^{m+l}\left\lceil\left\langle D_{i}^{S}, d\right\rangle\right\rceil+\#\left\{i:\left\langle D_{i}^{S}, d\right\rangle \in \mathbb{Z}_{<0}\right\}\right)} \prod_{i:\left\langle D_{i}^{S}, d\right\rangle \in \mathbb{Z}_{<0}} D_{i}+\text { h.o.t. },
\end{aligned}
$$

where

$$
\begin{aligned}
C_{d}= & \prod_{i:\left\langle D_{i}^{S}, d\right\rangle<0} \prod_{\left\langle D_{i}^{S}, d\right\rangle<k<0}\left(\left\langle D_{i}^{S}, d\right\rangle-k\right) \\
& \times \prod_{i:\left\langle D_{i}^{S}, d\right\rangle>0} \prod_{0 \leq k<\left\langle D_{i}^{S}, d\right\rangle}\left(\left\langle D_{i}^{S}, d\right\rangle-k\right)^{-1},
\end{aligned}
$$

The summand indexed by $d \in \mathbb{K}_{\mathrm{eff}, \mathcal{X}}$ contributes to the coefficient of $z^{-1}$ if and only if

$$
\sum_{i=1}^{m+l}\left\lceil\left\langle D_{i}^{S}, d\right\rangle\right\rceil+\#\left\{i:\left\langle D_{i}^{S}, d\right\rangle \in \mathbb{Z}_{<0}\right\} \leq 1 .
$$

This happens only in the following three cases:

- $\sum_{i=1}^{m+l}\left\lceil\left\langle D_{i}^{S}, d\right\rangle\right\rceil+\#\left\{i:\left\langle D_{i}^{S}, d\right\rangle \in \mathbb{Z}_{<0}\right\}=0$;

- $\left\{\begin{array}{l}\sum_{i=1}^{m+l}\left\lceil\left\langle D_{i}^{S}, d\right\rangle\right\rceil=0, \\ \#\left\{i:\left\langle D_{i}^{S}, d\right\rangle \in \mathbb{Z}_{<0}\right\}=1\end{array}\right.$ 
- $\left\{\begin{array}{l}\sum_{i=1}^{m+l}\left\lceil\left\langle D_{i}^{S}, d\right\rangle\right\rceil=1, \\ \#\left\{i:\left\langle D_{i}^{S}, d\right\rangle \in \mathbb{Z}_{<0}\right\}=0 .\end{array}\right.$

The first case happens if and only if $d=0$. If the second case happens, then

$$
\sum_{i=1}^{m+l}\left\lceil\left\langle D_{i}^{S}, d\right\rangle\right\rceil=\sum_{i=1}^{m+l}\left\langle D_{i}^{S}, d\right\rangle=\left\langle\rho_{\mathcal{X}}^{S}, d\right\rangle=0 .
$$

In particular,

$$
\left\langle D_{i}^{S}, d\right\rangle \in \mathbb{Z}, \quad 1 \leq i \leq m+l .
$$

Hence, we obtain the following lemma.

Lemma 4.7. For $1 \leq j \leq m+l$, the Batyrev element $\tilde{D}_{j}$ is given by

$$
\begin{gathered}
\tilde{D}_{j}=D_{j}+\sum_{i=1}^{m} D_{i} \sum_{\substack{\left\langle\rho_{\mathcal{X}}^{S}, d\right\rangle=0 \\
\left\langle D_{i}^{S}, d\right\rangle \in \mathbb{Z}_{<0} \\
\left\langle D_{k}^{S}, d\right\rangle \in \mathbb{Z}_{\geq 0}, \forall k \neq i}} C_{d}\left\langle D_{j}^{S}, d\right\rangle y^{d} \\
+\sum_{\substack{\sum_{i=1}^{m+l}\left\lceil\left\langle D_{i}^{S}, d\right\rangle\right\rceil=1 \\
\left\langle D_{i}^{S}, d\right\rangle \notin \mathbb{Z}_{<0}, \forall i}} C_{d}\left\langle D_{j}^{S}, d\right\rangle y^{d} \mathbf{1}_{v(d)},
\end{gathered}
$$

where $C_{d}$ is given by equation (46).

\section{Seidel Elements Corresponding to Box Elements}

Consider the box element $s_{j} \in \operatorname{Box}(\Sigma)$ such that

$$
\bar{s}_{j}=\sum_{i=1}^{m} c_{j i} \bar{b}_{i} \in \mathbf{N}_{\mathbb{Q}} \quad \text { for some } 0 \leq c_{j i}<1 .
$$

Note that $c_{j i}=0$ for $i \in I_{j}^{S}$. We define a $\mathbb{C}^{\times}$-action on $\mathcal{U}^{S} \times\left(\mathbb{C}^{2} \backslash\{0\}\right)$ by

$$
\left(z_{1}, \ldots, z_{m+l}, u, v\right) \mapsto\left(z_{1}, \ldots, t^{-1} z_{m+j}, \ldots, z_{m+l}, t u, t v\right), \quad t \in \mathbb{C}^{\times} .
$$

Hence, we have the associated bundle

$$
\mathcal{E}_{m+j}=\mathcal{U}^{S} \times\left(\mathbb{C}^{2} \backslash\{0\}\right) / G^{S} \times \mathbb{C}^{\times}
$$

over $\mathbb{C P}^{1}$ with fiber $\mathcal{X}$.

We can identify $H^{2}\left(\mathcal{E}_{m+j} ; \mathbb{Q}\right)$ with $H^{2}(\mathcal{X} ; \mathbb{Q}) \oplus \mathbb{Q}$ using the section $\left[\sigma_{0}\right]$. Note that this splitting given by the section $\left[\sigma_{0}\right]$ of $\mathcal{E}_{m+j}$ does not give a splitting of $\operatorname{Pic}\left(\mathcal{E}_{m+j}\right)$ over $\mathbb{Z}$ since the intersection numbers of $\left[\sigma_{0}\right]$ with line bundles on $\mathcal{E}_{m+j}$ are fractional.

The fan of $\mathcal{E}_{m+j}$ is contained in $N_{\mathbb{Q}} \oplus \mathbb{Q}$. The 1 -skeleton is given by

$$
\hat{b}_{i}=\left(b_{i}, 0\right), \quad \text { for } 1 \leq i \leq m ; \quad \hat{b}_{m+1}=(0,1) ; \quad \hat{b}_{m+2}=\left(s_{j},-1\right) .
$$

Then

$$
\hat{D}_{i}^{S}=\left(D_{i}^{S},-c_{j i}\right), \quad \text { for } 1 \leq i \leq m \text {; }
$$




$$
\begin{aligned}
\hat{D}_{m+i}^{S} & =\left(D_{m+i}^{S}, 0\right) \quad \text { for } 1 \leq i \leq l ; \\
\hat{D}_{m+l+1}^{S} & =\hat{D}_{m+l+2}^{S}=(0,1) .
\end{aligned}
$$

The Seidel element is defined as in equation (5). Moreover, we define $\left\{p_{0}, p_{1}\right.$, $\left.\ldots, p_{r}\right\}$ and $\left\{p_{0}^{S}, p_{1}^{S}, \ldots, p_{r+l}^{S}\right\}$ in the same way as in Section 3.2.

As in the toric divisor case, we have the following expansion of the $I$-function:

$$
\begin{aligned}
I_{\mathcal{E}_{m+j}}(y, z)= & e^{\sum_{i=0}^{r} p_{i} \log y_{i} / z}\left(1+z^{-1}\left(\sum_{i=0}^{r} g_{i}^{(m+j)}(y) p_{i}+\tau_{t w}^{(m+j)}(y)\right)\right. \\
& \left.+z^{-2}\left(\sum_{n=0}^{2} G_{n}^{(m+j)}(y) y_{0}^{n}\right)+O\left(z^{-3}\right)\right),
\end{aligned}
$$

and using the same argument as in Lemmas 3.13 and 3.15, we can show that $g_{i}^{(m+j)}(y)$ and $\tau_{t w}^{(m+j)}(y)$ are independent from $y_{0}$ for $1 \leq i \leq r$ and $1 \leq j \leq l$. Moreover, for each $j \in\{1, \ldots, l\}$, we have

$$
g_{i}^{(m+j)}\left(y_{0}, \ldots, y_{r+l}\right)=g_{i}\left(y_{1}, \ldots, y_{r+l}\right) \quad \text { for } i=1, \ldots, r
$$

and

$$
\iota^{*} \tau_{t w}^{(m+j)}(y)=\tau_{t w}(y) .
$$

We also obtain the following theorem.

TheOREM 5.1. The Seidel element $\tilde{S}_{m+j}$ associated to the box element $s_{j}$ is given by

$$
\tilde{S}_{m+j}(\tau(y)):=\tilde{S}_{m+j}\left(\tau^{(m+j)}(y)\right)=\exp \left(-g_{0}^{(m+j)}(y)\right) \iota^{*}\left(G_{1}^{(m+j)}(y)\right) .
$$

Using the same computation as in the toric divisor case, we can compute the correction coefficient $g_{0}^{(m+j)}$.

Lemma 5.2. The function $g_{0}^{(m+j)}$ is given by

$$
=\sum_{1 \leq k \leq m, k \notin I_{j}^{S}} \sum_{\begin{array}{r}
\left\langle D_{i}^{S}, d\right\rangle \in \mathbb{Z}, 1 \leq i \leq m+l \\
\left\langle\rho_{\mathcal{X}}^{S}, d\right\rangle=0 \\
\left\langle D_{k}^{S}, d\right\rangle<0 \\
\left\langle D_{i}^{S}, d\right\rangle \geq 0, \forall i \neq k
\end{array}} c_{j k} \frac{(-1)^{-\left\langle D_{k}^{S}, d\right\rangle}\left(-\left\langle D_{k}^{S}, d\right\rangle-1\right) !}{\prod_{i \neq k}\left\langle D_{i}^{S}, d\right\rangle !} y^{d},
$$

where $I_{j}^{S}$ is the "anticone" of the cone containing $s_{j}$.

Proof. The argument is almost the same as the argument in Section 3.5. The only change we need to make is the paragraph before Lemma 3.17:

In this case, $g_{0}^{(m+j)}$ is the coefficient corresponding to $p_{0}$, and elements in $\left\{\hat{D}_{1}, \ldots, \hat{D}_{m}\right\}$ that contain $p_{0}$ are precisely the following elements:

$$
\hat{D}_{k}=\left\langle D_{k},-c_{j k}\right\rangle=D_{k}-c_{j k} p_{0} \quad \text { for } 1 \leq k \leq m \text { and } k \notin I_{j}^{S} .
$$


Therefore, by expressions (37) and (39) we must have $\left\langle D_{k}^{S}, d\right\rangle<0$ for exactly one $k$ in $\left\{k \in \mathbb{Z} \mid 1 \leq k \leq m\right.$ and $\left.k \notin I_{j}^{S}\right\}$.

Moreover, by mimicking the computation in Lemma 4.4 we have the following:

LEMma 5.3. The I-function of $\mathcal{E}_{m+j}$ satisfies the following differential equation:

$$
z \frac{\partial}{\partial y_{0}}\left(y_{0} \frac{\partial}{\partial y_{0}}\right) I_{\mathcal{E}_{j}}=y^{-D_{m+j}^{S \vee}}\left(\sum_{i=1}^{r+l} m_{i j}\left(y_{i} \frac{\partial}{\partial y_{i}}\right)-y_{0} \frac{\partial}{\partial y_{0}}\right) I_{\mathcal{E}_{j}},
$$

where $D_{m+j}^{S \vee} \in \mathbb{L}^{S} \otimes \mathbb{Q}$ is defined by (18).

Proof. The proof is almost identical to the proof of Lemma 4.4, except, this time, we need to choose $\beta=\left[\sigma_{0}\right]-D_{m+j}^{S \vee}$. Then everything else follows.

Using the lemmas and following the argument in the toric divisor case, we conclude:

TheOREM 5.4. The Seidel element $\tilde{S}_{m+j}$ corresponding to the box element $s_{j}$ with

$$
\bar{s}_{j}=\sum_{i=1}^{m} c_{j i} \bar{b}_{i} \quad \text { for some } 0 \leq c_{j i}<1
$$

is given by

$$
\tilde{S}_{m+j}\left(\tau^{(m+j)}(y)\right)=\exp \left(-g_{0}^{(m+j)}\right) y^{-D_{m+j}^{S \vee}} \tilde{D}_{m+j}(y),
$$

where $\tilde{D}_{m+j}(y)$ is the corresponding Batyrev element. Moreover,

$$
\begin{aligned}
\tilde{D}_{m+j}= & \sum_{i=1}^{m} D_{i} \sum_{\substack{\left\langle\rho_{\mathcal{X}}^{S}, d\right\rangle=0 \\
\left\langle D_{i}^{S}, d\right\rangle \in \mathbb{Z}_{<0} \\
\left\langle D_{k}^{S}, d\right\rangle \in \mathbb{Z}_{\geq 0}, \forall k \neq i}} C_{d}\left\langle D_{m+j}^{S}, d\right\rangle y^{d} \\
& +\sum_{\substack{\sum_{i=1}^{m+l}\left\lceil\left\langle D_{i}^{S}, d\right\rangle\right\rceil=1 \\
\left\langle D_{i}^{S}, d\right\rangle \notin \mathbb{Z}_{<0}, \forall i}} C_{d}\left\langle D_{m+j}^{S}, d\right\rangle y^{d} \mathbf{1}_{v(d)},
\end{aligned}
$$

and

$$
\begin{aligned}
C_{d}= & \prod_{i:\left\langle D_{i}^{S}, d\right\rangle<0} \prod_{\left\langle D_{i}^{S}, d\right\rangle<k<0}\left(\left\langle D_{i}^{S}, d\right\rangle-k\right) \\
& \times \prod_{i:\left\langle D_{i}^{S}, d\right\rangle>0} \prod_{0 \leq k<\left\langle D_{i}^{S}, d\right\rangle}\left(\left\langle D_{i}^{S}, d\right\rangle-k\right)^{-1} .
\end{aligned}
$$

ACKNOWLEDGMENTS. The author wants to thank professor Hsian-Hua Tseng for his guidance and lots of helpful discussions. The author is also very grateful for the referees' numerous useful comments and suggestions. 


\section{References}

[1] V. Batyrev, Quantum cohomology rings of toric manifolds, Astérisque 218 (1993), 9-34. Journées de Géométrie Algébrique d'Orsay (Orsay, 1992).

[2] L. Borisov, L. Chen, and G. Smith, The orbifold Chow ring of toric Deligne-Mumford stacks, J. Amer. Math. Soc. 18 (2005), no. 1, 193-215.

[3] K. Chan, C.-H. Cho, S.-C. Lau, and H.-H. Tseng, Gross fibrations, SYZ mirror symmetry, and open Gromov-Witten invariants for toric Calabi-Yau orbifolds, arXiv:1306.0437 [math.SG].

[4] T. Coates, A. Corti, H. Iritani, and H.-H. Tseng, A mirror theorem for toric stacks, arXiv:1310.4163 [math.AG].

[5] E. Gonzalez and H. Iritani, Seidel elements and mirror transformations, Selecta Math. (N.S.) 18 (2012), no. 3, 557-590.

[6] H. Iritani, An integral structure in quantum cohomology and mirror symmetry for toric orbifolds, Adv. Math. 222 (2009), 1016-1079.

[7] Y. Jiang, The orbifold cohomology ring of simplicial toric stack bundles, Illinois J. Math. 52 (2008), no. 2, 493-514.

[8] D. McDuff, Quantum homology of fibrations over $S^{2}$, Internat. J. Math. 11 (2000), 665-721.

[9] D. McDuff and S. Tolman, Topological properties of Hamiltonian circle actions, IMRP Int. Math. Res. Pap. 72826 (2006), 1-77.

[10] P. Seidel, $\pi_{1}$ of symplectic automorphism groups and invertibels in quantum homology rings, Geom. Funct. Anal. 7 (1997), 1046-1095.

[11] H.-H. Tseng and D. Wang, Seidel representation for symplectic orbifolds, arXiv:1207.4246.

Department of Mathematics

The Ohio State University

100 Math Tower

231 West 18th Avenue

Columbus, $\mathrm{OH} 43210$

USA

you.111@osu.edu 